\title{
Outer structure of the Galactic warp and flare: explaining the Canis Major over-density ${ }^{\star}$
}

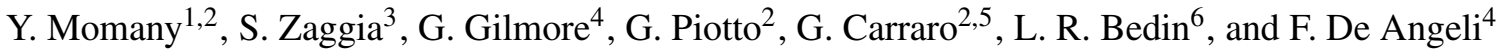 \\ ${ }^{1}$ INAF- Oss. Astronomico di Padova, Vicolo dell'Osservatorio 5, 35122 Padova, Italy \\ e-mail: yazan.almomany@oapd.inaf.it \\ 2 Dip. di Astronomia, Università di Padova, Vicolo dell'Osservatorio 2, 35122 Padova, Italy \\ e-mail: momany, piotto@pd.astro.it \\ 3 INAF - Oss. Astronomico di Trieste, Via Tiepolo 11, 34131 Trieste, Italy \\ e-mail: zaggia@oats.inaf.it \\ ${ }^{4}$ Institute of Astronomy, University of Cambridge, Cambridge, CB3 OHA, UK \\ e-mail: gil,fda@ast.cam.ac.uk \\ 5 Andes Fellow, Departamento de Astronomía, Universidad de Chile, Casilla 36-D, Santiago, Chile \\ Astronomy Department, Yale University, New Haven, CT 06511, USA \\ e-mail: gcarraro@das.uchile.cl \\ ${ }^{6}$ European Southern Observatory, Karl-Schwarzschild-Str. 2, 85748 Garching b. München, Germany \\ e-mail: 1bedin@eso.org
}

Received 21 August 2005 / Accepted 28 February 2006

\section{ABSTRACT}

Aims. In this paper we derive the structure of the Galactic stellar warp and flare. Methods. We use 2MASS red clump and red giant stars, selected at mean and fixed heliocentric distances of $R_{\odot} \simeq 3,7$ and $17 \mathrm{kpc}$. Results. Our results can be summarized as follows: (i) a clear stellar warp signature is derived for the 3 selected rings, proving that the warp starts already within the solar circle; (ii) the derived stellar warp is consistent (both in amplitude and phase-angle) with that for the Galactic interstellar dust and neutral atomic hydrogen; (iii) the consistency and regularity of the stellar-gaseous warp is traced out to about $R_{\mathrm{GC}} \sim 20 \mathrm{kpc}$; (iv) the Sun seems not to fall on the line of nodes. The stellar warp phase-angle orientation $\left(\phi \sim 15^{\circ}\right)$ is close to the orientation angle of the Galactic bar and this, most importantly, produces an asymmetric warp for the inner $R_{\odot} \simeq 3$ and $7 \mathrm{kpc}$ rings; (v) a Northern/Southern warp symmetry is observed only for the ring at $R_{\odot} \simeq 17 \mathrm{kpc}$, at which the dependency on $\phi$ is weakened; (vi) treating a mixture of thin and thick disk stellar populations, we trace the variation with $R_{\mathrm{GC}}$ of the disk thickness (flaring) and derive an almost constant scale-height ( $\left.\sim 0.65 \mathrm{kpc}\right)$ within $R_{\mathrm{GC}} \sim 15 \mathrm{kpc}$. Further out, the disk flaring increase gradually reaching a mean scale-height of $\sim 1.5 \mathrm{kpc}$ at $R_{\mathrm{GC}} \sim 23 \mathrm{kpc}$; (vii) the derived outer disk warping and flaring provide further robust evidence that there is no disk radial truncation at $R_{\mathrm{GC}} \sim 14 \mathrm{kpc}$.

Conclusions. In the particular case of the Canis Major (CMa) over-density we confirm its coincidence with the Southern stellar maximum warp occurring near $l \sim 240^{\circ}$ (for $R_{\odot} \simeq 7 \mathrm{kpc}$ ) which brings down the Milky Way mid-plane by $\sim 3^{\circ}$ in this direction. The regularity and consistency of the stellar, gaseous and dust warp argues strongly against a recent merger scenario for Canis Major. We present evidence to conclude that all observed parameters (e.g. number density, radial velocities, proper motion etc) of CMa are consistent with it being a normal Milky Way outer-disk population, thereby leaving no justification for more complex interpretations of its origin. The present analysis or outer disk structure does not provide a conclusive test of the structure or origin of the Monoceros Ring. Nevertheless, we show that a warped flared Milky Way contributes significantly at the locations of the Monoceros Ring. Comparison of outer Milky Way H I and CO properties with those of other galaxies favors the suggestion that complex structures close to planar in outer disks are common, and are a natural aspect of warped and flaring disks.

Key words. Galaxy: structure - Galaxy: formation - galaxies: interactions - galaxies: individual: Canis Major

\section{Introduction}

There was a revitalisation of interest in the outer disk of the Milky Way with the photometric discovery of significant over-

^ Based on archival data collected at the European Southern Observatory, La Silla, Chile. densities of F stars in the Sloan Digital Sky Survey by Newberg et al. (2002) towards the Galactic anti-center. The reported over-densities defined a type of stellar Ring structure, relatively confined to the Galactic plane $\left(|b| \leq 30^{\circ}\right)$, and stretching over the sky for $\sim 100^{\circ}$ in longitude centered in Monoceros. A kinematic and spectroscopic study of the Ring structure by 
Yanny et al. (2003) confirmed (i) a Galactocentric distance of 18 and $20 \mathrm{kpc}$ (respectively above and below the Galactic plane); (ii) an inconsistency of its velocity dispersion with typical Galactic halo and thick disk structures; and (iii) a metallicity of $[\mathrm{Fe} / \mathrm{H}] \simeq-1.6$ consistent with halo populations. In the context of continuing searches for outer structure related to the Sgr dwarf, and other possible accretion events the most natural scenario proposed by Yanny etal for the Monoceros Ring (Mon. Ring) was that it traced a remnant dwarf satellite galaxy in the late process of disruption (if not already dissolved). Accumulating evidence supporting the existence of the Mon. Ring (Rocha-Pinto et al. 2003; Crane et al. 2003; Frinchaboy et al. 2004) soon triggered a search for a progenitor, obviously carried out near the Galactic plane.

At the same time, in an alternative, more conservative scenario, the existence of the Mon. Ring was seen as the consequence of perturbations in the outer disk caused by ancient warps (Ibata et al. 2003). Indeed, the Mon. Ring rotates in a prograde orbit that is almost circularized, strongly suggestive of a disk origin. Nevertheless, simulations by Helmi (2003) showed that accretion models, where co-planar streams can follow circular orbits, were indeed feasible. This encouraged a search for a progenitor of the Ring: if there is one (the Sagittarius dwarf) why not another.

In an analysis of 2MASS data, Martin et al. (2004a) assumed a symmetric Galactic vertical stellar distribution around $b=0^{\circ}$ and searched for asymmetrical differences between Northern and Southern star-counts. Among other features, they pointed to an elliptical-shaped stellar over-density centered at $(l, b)=\left(240^{\circ},-7^{\circ}\right)$. They interpreted this over-density as the core of a satellite galaxy currently undergoing in-plane accretion, namely the Canis Major (CMa) dwarf spheroidal galaxy, the best Ring progenitor candidate.

In Momany et al. (2004b), we highlighted the fact that in the analyses in Martin et al. (2004a) and Bellazzini et al. (2004, first astro-ph version) the possible influence of a Galactic stellar warp on the detection of a vertically asymmetric distribution so close to the Galactic plane was not considered. Observationally, the warp is a bending of the Galactic plane upwards in the first and second Galactic longitude quadrants $\left(0^{\circ} \leq l \leq 180^{\circ}\right)$ and downward in the third and fourth quadrants $\left(180^{\circ} \leq l \leq 360^{\circ}\right)$. We emphasise that the suggested reality of an outer stellar warp was not a new proposal by us. Among other earlier studies, one of particular relevance is that of Carney \& Seitzer (1993) who analysed the "Galaxy's own structure to obtain at least a peak at the outer disk", i.e. using certain lines of sights, one can look away from the plane, reducing the foreground disk signal as well as reddening-absorption-crowding and derive the age and metallicity of the outer warped disk. Analyzing the color-magnitude diagrams of fields very near to the CMa center $\left[(l, b)=\left(245^{\circ},-4^{\circ}\right)\right]$ Carney \& Seitzer claimed to have detected the main sequence and turnoff region of the outer Galactic disk. On the other hand, the analysis of Martin et al. (2004a) and Bellazzini et al. (2004) discounted the Galactic warp in this zone, so that the stellar populations previously identified as outer disk main sequence, were now proposed as an un-expected stellar population. Further hints of a starcount anomaly at $l=240^{\circ}$ are found in Alard (2000) who, again, associated the "strong asymmetry" in this region with the Galactic stellar warp.

In Momany et al. (2004b) we concluded that the CMa over-density can be fully accounted for if the Galactic disk (at $l=240^{\circ}$ ) is $2^{\circ}$ displaced/warped below the mid-plane; i.e. the symmetry axis for this region is at $b=-2^{\circ}$ and not $b=0^{\circ}$. In response to our analysis, Martin et al. (2004b) presented radial velocities and argued that the Galactic stellar warp (location and amplitude), cannot explain the CMa over-density. In particular, they argued that (i) a warp angle of $-2^{\circ}$ is not enough to erase the CMa over-density; and (ii) the CMa over-density is stronger in amplitude and located too far from the Southern hemisphere warp at $l=270^{\circ}$. More recently, Rocha-Pinto et al. (2005) proposed that the amplitude of the CMa over-density is small with respect to another over-density, this time located in Argo $\left(l \sim 290^{\circ}\right)$. In this later analysis, Rocha-Pinto et al. view the CMa over-density as the consequence of a dust extinction window aligned with a maximum warp location at $l=245^{\circ}$ (as seen in López-Corredoira et al. 2002). Confusingly, Martin et al. (2004b) and Bellazzini et al. (2006) use the same source (López-Corredoira et al. 2002) to argue that the maximum warp location is at $l=270^{\circ}$.

In order to clarify this situation, it is timely to re-evaluate the detailed properties of the Galactic stellar warp, specifically the location and amplitude of its maximum. Most recently, Conn et al. (2005) presented a wide-field survey of the Mon. Ring and emphasised that the presence of Ring streams above and below the Galactic plane argue against a Galactic origin of the Mon. Ring. Moreover, they suggested that positive detections of the Mon. Ring below the plane may also be correlated with the Triangulum-Andromeda(TriAnd) structure (Majewski et al. 2004; Rocha-Pinto et al. 2005). In this scenario, although the Mon. Ring and TriAnd structure are located at different distances, the TriAnd structure could be the distant arm of a multiply-wrapped tidal stream.

In the main part of this paper we use 2MASS data to derive and trace the signature of the Galactic stellar warp as a function of Galactic longitude. Our working hypothesis is simple: had the CMa over-density been due to an extra-Galactic accretion, one would expect it to appear as a distortion on top of a large-scale structure, that is the Galactic stellar warp. As we shall demonstrate, CMa as an over-density is easily accounted for as being the maximum Southern stellar warp. This, in our opinion, allows Occam's Razor to indicate a clear preference for a Galactic structural origin of the CMa over-density. We then discuss evidence which we believe associates the Mon. stream with the warped and flaring Galactic disk. In several Appendices we briefly review and comment on important aspects of the observational properties of CMa and the Mon Ring.

\section{The Galactic stellar warp}

In Bellazzini et al. (2006) the authors commented on how the different adopted parametrization of the warp may lead to some confusion. Indeed, the warp has been derived from: (1) the mean latitude of the adopted tracer as a function of longitude (Djorgovski \& Sosin 1989); (2) the ratio of star counts in Northern and Southern hemispheres as a function of 
longitude (López-Corredoira et al. 2002); and (3) the latitude of peak brightness as a function of longitude (Freudenreich et al. 1994). There has been some disagreement about these determinations. Bellazzini et al. (2006) argued that the Momany et al. parametrization of the warp was "not a fair description" of the South/North over-densities. In the following we will firstly expand on our method of tracing the Galactic warp and then secondly we will compare our results with those derived by other methods. This comparison show excellent consistency.

In Momany et al. (2004b, Fig. 2) we extracted dereddened 2MASS M-giants $\left(0.85 \leq(J-K)_{0} \leq 1.3\right)$ within an oblique box surrounding the CMa red giant branch in the CMD, and falling in a strip between $-20^{\circ} \leq b \leq 20^{\circ}$ and $235^{\circ} \leq l \leq 245^{\circ}$. Initially assuming reflection symmetry around $b=0^{\circ}$ (warp angle $w=0^{\circ}$ ) we performed Northern and Southern star counts, binned in $0.5^{\circ}$. This showed clearly (upper panels of the same figure) that by assuming a symmetry around $b=0^{\circ}$, as done in Martin et al. (2004a), one can recover the identified (CMa) over-density. Successively the symmetry axis was varied within the range $|b| \leq 5^{\circ}$, in steps of $0.1^{\circ}$. For each step (i.e. each warp angle) the Northern and Southern latitude profiles were folded and a reduced $\chi^{2}$ defined as:

$\chi^{2}=\sum\left[\left(y_{\text {North }}-y_{\text {South }}\right)^{2} /\left(y_{\text {North }}+y_{\text {South }}\right)\right]$

was employed to determine the warp angle which minimized the differences between the two profiles $(y$ is the $\log (N)$ of star counts computed in the latitude profiles, see Fig. 4). A symmetry of around $b=-2^{\circ}$ (i.e warp angle $w=-2^{\circ}$ ) almost completely canceled the (CMa) over-density. This warp angle has been derived (not modeled as argued in Martin et al. (2004a) and Bellazzini et al. (2004)) and agrees with the angle derived for the gas warp by Freudenreich (1994). It is important to note that this result is obtained independently of the adopted dereddening method: (i) pure Schlegel et al. (1998) values; or (ii) modified with the Bonifacio et al. (2000) correction (see Amôres \& Lépine (2005) for recent confirmation of errors in the Schlegel et al. values).

Thus, we are able to measure the latitude angle at which the latitude profiles crossing the Galactic disk are symmetric; i.e. the warp-angle or the mid-plane of the warped disk. In this section we apply this method for the entire 2MASS catalog within $-20^{\circ} \leq b \leq 20^{\circ}$, and derive the global Galactic warp as a function of longitude. Most importantly, the warp signature is traced by means of different stellar sources (red clump (RC) and red giants (RGB)) at different distances. This is particularly important because we want to investigate the impact of the tracer contamination and its distance on the derived warp signature.

To emphasize the particular importance of the contamination and distance properties of the assumed tracer (and the intrinsic difficulty for similar investigations) we will briefly comment on two recent studies probing the Galactic warp, namely Yusifov (2004) and López-Corredoira et al. (2002).

\subsection{The Yusifov stellar warp model}

In his analysis, Yusifov used the asymmetric distribution of 1412 Galactic pulsars (from the Manchester et al. 2005

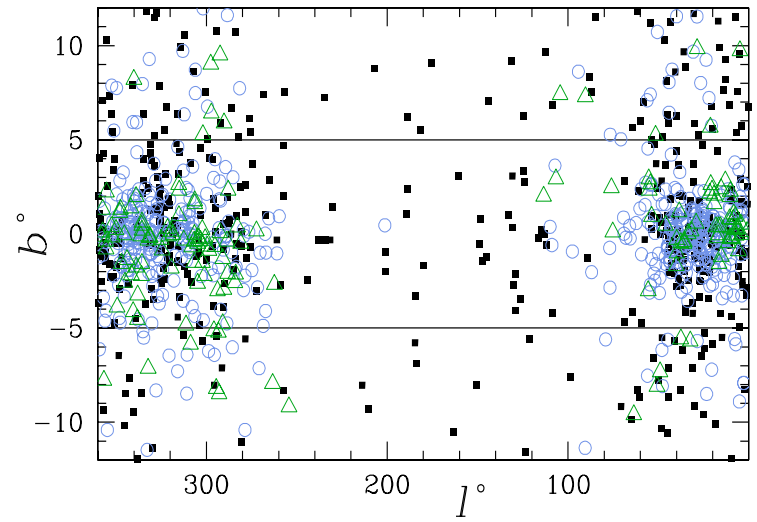

Fig. 1. The distribution pulsars in the Manchester et al. (2005) catalog. Filled squares and open circles are pulsars with Galactocentric distances between 1 and $5 \mathrm{kpc}$, and 5 and $10 \mathrm{kpc}$ respectively. Open triangles are pulsars at $R_{\mathrm{GC}} \geq 10 \mathrm{kpc}$.

catalog) to trace the Galactic stellar warp and flare (the increase in scale-height as a function of Galactocentric distance). Yusifov limited his analysis to pulsars within $|b| \leq 5^{\circ}$ whose distances are $\geq 1 \mathrm{kpc}$. He calculated the ratio of the cumulative number of pulsars above and below the Galactic plane as a function of Galactic longitude, and so derived a warp model. However, as seen in Fig. 1, the Manchester et al. (2005) cata$\log$ has only 1 pulsar at $R>5 \mathrm{kpc}$ between $200^{\circ} \leq l \leq 270^{\circ}$. Clearly, the pulsar catalog is already strongly incomplete at $R \sim 5 \mathrm{kpc}$ in the outer Milky Way. Although one might doubt how these data can reliably predict the number density of stars at CMa distances, it is true that the Yusifov warp model predictions have turned out to be comparable with other studies based on more complete samples, and we therefore will compare our results with this model.

\subsection{The López-Corredoira et al. stellar warp model}

The López-Corredoira et al. 2MASS-based investigation was a major attempt at studying the Galactic stellar warp and flare. There are, however, a few points that must be kept in mind regarding their analysis. Firstly, 2MASS was not complete at the time that their analysis was concluded, so that they were able to analyse only 820 lines of sight, each of area between 0.5 and 1.0 square degrees, restricted to $|b|= \pm 3^{\circ}, \pm 6^{\circ}, \pm 9^{\circ}$. This is particularly important in understanding the determination (cf. Martin et al. 2004b; Bellazzini et al. 2004, 2006) of the maximum of the stellar warp at $l \sim 270^{\circ}$, a result often cited from the López-Corredoira et al. paper. Indeed, the area around $l \sim 270^{\circ}$ was missing in the López-Corredoira et al. study, and nowhere in that article is it stated that the stellar warp maximum is at $l \sim 270^{\circ}$. Indeed the formula describing the López-Corredoira et al. warp model at $\mathrm{CMa}$ distances shows that the maximum stellar warp is near to $l \sim 240^{\circ}$ (see Sect. 4). The value of $l \sim 240^{\circ}$ for the maximum Galactic warp is in fact found in other studies, e.g. Freudenreich (1994, their Fig. 3) studying 


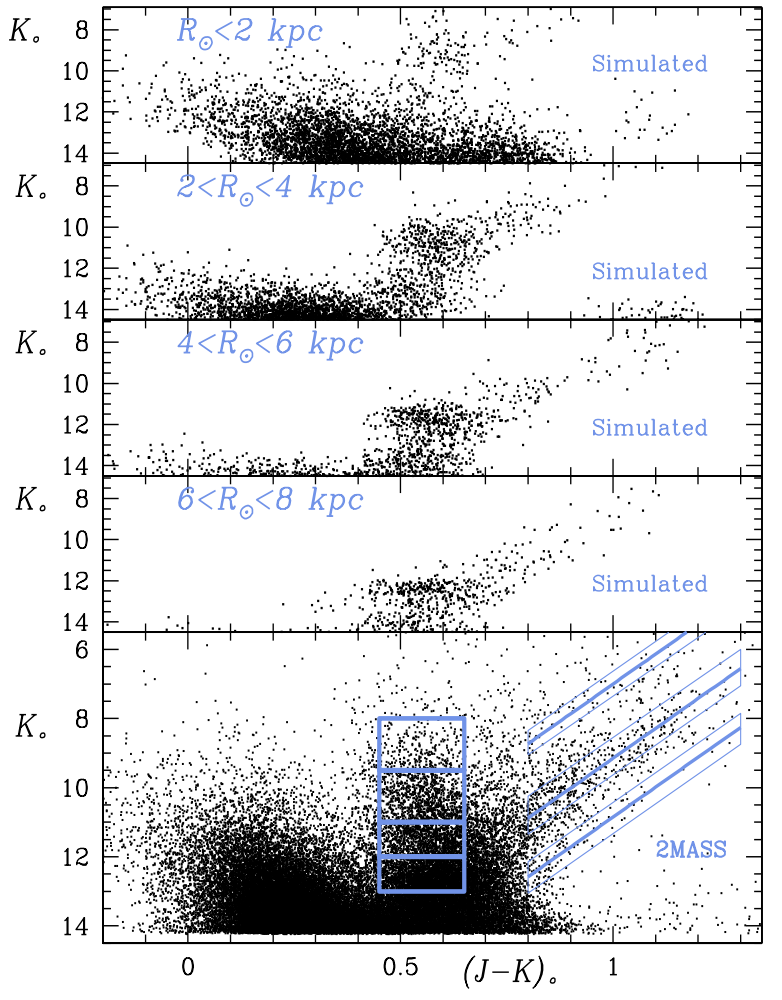

Fig. 2. The upper 4 panels display a Besançon $1^{\circ}$ square area simulated color-magnitude diagram around $\mathrm{CMa}(l, b)=\left(240^{\circ},-7^{\circ}\right)$. These show the expected different contributions of stars as a function of their distance. The lowest panel displays data from 2MASS between $238^{\circ} \leq l \leq 242^{\circ}$ and $-8^{\circ} \leq b \leq-6^{\circ}$. The outlined vertical boxes mark 4 distance intervals as selected from the red clump distribution in simulated diagrams. The 3 oblique boxes trace 3 samples of red giant stars extracted at three different heliocentric distances. The thick line is the Majewski et al. calibration of the Sagittarius dwarf RGB, shifted to $2.8,7.3$ and $16.6 \mathrm{kpc}$.

the gaseous warp, and Djorgovski \& Sosin (1989, their Fig. 1) analyzing IRAS sources ${ }^{1}$.

Second, López-Corredoira et al. derive the ratio of Northern to Southern star-counts, $R_{\mathrm{RC}}=N_{\text {North }} / N_{\text {South }}$ for red clump stars with $K_{0} \leq 14.0$ (see their Fig. 15). To help us evaluate the impact of contamination when using RC stars as warptracers, in Fig. 2 we use Besançon (Robin et al. 2003) simulated color-magnitude diagrams around CMa and plot the data as a function of their distance. Focusing our attention around $(J-K)_{0} \simeq 0.6$, one sees that the stellar populations at $\leq 2 \mathrm{kpc}$ which will contribute to the final $R_{\mathrm{RC}}^{K \leq 14.0}$ ratio include: (i) $\mathrm{RC}$ stars at $8.0 \leq K \leq 10.0$, and most importantly, (ii) dwarfs at the fainter magnitudes $K_{0} \geq 11.5$. At magnitudes around the $\mathrm{RC}$ of $\mathrm{CMa}(K \sim 13.0)$, we estimate a contamination by local foreground dwarfs of about 25-30\%. Thus, if one is interested in the $R_{\mathrm{RC}}$ ratio at say, the CMa distance, one must bear in mind that this ratio is subject to potentially serious contamination by RC and dwarf stars at distances closer than the CMa over-density.

${ }^{1}$ In particular note a clear warp excess around $l \sim 240^{\circ}$ with respect to their fitted function.

\subsection{Tracing the stellar warp and flare with $R C$ and RGB stars}

We employ our method in tracing the Galactic warp and flare using two stellar tracers: red clump and red giant stars. For both tracers we repeat the analysis for different mean distances. The advantage in using two stellar tracers derives from their different contamination status. With respect to RC stars, red giants are an ideal instrument in probing the Galactic warp and flare since these suffer less external contamination by nearby dwarfs. At the same time red giants projected at different distances do not overlap in the color-magnitude diagram (i.e. no internal contamination) and this guarantees a better distance separation. Indeed, being the bright and evolved part of the faint and un-detected main sequence stars, red giants allow us (already in the not so deep 2MASS catalog) to probe the most distant, and almost entire, Milky Way disk populations.

The vertical boxes in Fig. 2 set 4 heliocentric distance intervals $(\leq 2,2 \div 4,4 \div 6$ and $6 \div 8 \mathrm{kpc})$ from which we extract $\mathrm{RC}$ stars and use their star-counts to derive the warp and flare.

Similarly, the 3 oblique boxes trace red giant stars at fixed heliocentric distances between $2.3 \div 3.2,5.5 \div 9.1$, and $13.2 \div 20.0 \mathrm{kpc}$. Thus, the mean assumed distances of the RGB samples are 2.8, 7.3 and $16.6 \mathrm{kpc}$, with the intermediate sample being centered on the CMa RGB. To estimate the distances to Milky Way M-giants we apply the same method as in Martin et al. (2004a), that first led to the identification of the CMa over-density. This is done by using the Sagittarius dwarf RGB as a reference. Assuming a distance modulus of 16.9 and a mean $[\mathrm{Fe} / \mathrm{H}]$ of $\sim-0.5^{2}$ Majewski et al. (2003) derived the following calibration of the Sagittarius RGB: $K=$ $-8.650 \times(J-K)_{0}+20.374$ (see the thick line in Fig. 2). The mean metallicity of Sagittarius can be considered intermediate between inner (more metal-rich) and outer (more metal-poor) Milky Way disk stars. Indeed, the mean abundance of the disk stars is known to vary between $-1.0 \leq[\mathrm{Fe} / \mathrm{H}] \leq+0.3$ (see Bensby et al. (2004) for differences between thin and thick disk populations)

At the same time we note that the Majewski et al. RGB calibration would reproduce the entire range of disk metallicities if age is allowed to vary. For example the Sagittarius RGB would overlap with a $10 \mathrm{Gyr}$ and $[\mathrm{Fe} / \mathrm{H}]=-0.7$ theoretical isochrone (appropriate for outer disk populations?) as well as one with $4 \mathrm{Gyr}$ and $[\mathrm{Fe} / \mathrm{H}]=-0.4$ (appropriate for inner disk populations?). A systematic uncertainty in estimating the distances of the Galactic M-giants is therefore un-avoidable. Similarly, contamination between different populations and uncertainties in reddening corrections will cloud our analysis. A schematic view of the Milky Way and of the regions where we probe the stellar warp and flare are shown in Fig. 3.

Examples of the application of our method are shown in the upper horizontal panels of Fig. 4, reporting the derivation of the best warp angle for two fields extracted from the $R_{\odot}=$ $7.3 \mathrm{kpc}$ RGB sample. We show two cases: the first field $80^{\circ} \leq$ $l \leq 90^{\circ}$ and $|b| \leq 20^{\circ}$ is more populated and the warp angle

\footnotetext{
${ }^{2}$ A recent study by Monaco et al. (2005) report on high resolution UVES spectroscopy showing a mean metallicity of $[\mathrm{Fe} / \mathrm{H}]=-0.41 \pm 0.20$.
} 


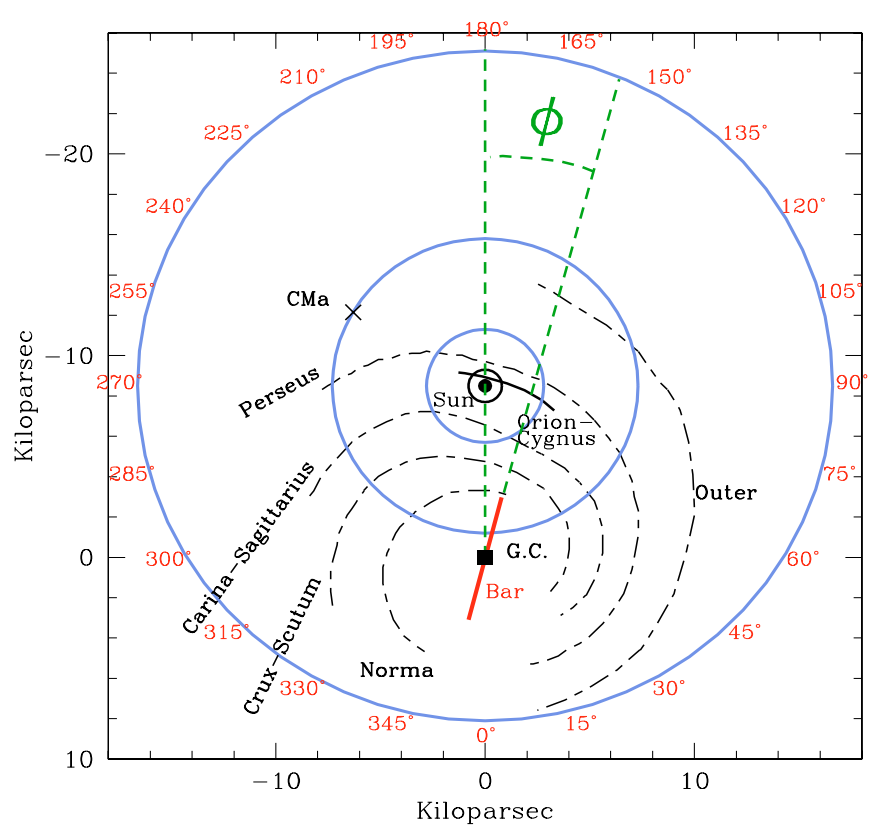

Fig. 3. A schematic view (from Drimmel \& Spergel 2001) of the Milky Way as seen from its North pole showing the 4 spiral arms as mapped by H II regions and dust (see also Vallée 2005; and Russeil 2003). The Galactic center, the Sun and the names of the spiral arms are plotted. Note the presence of the Local arm (Orion-Cygnus) close to the Sun's position and the outer arm that is also called Norma-Cygnus. The 3 heliocentric circles define the regions at fixed $R_{\odot}$ where we extract RGB samples to trace the Galactic warp and flare. The direction of the warp phase angle $\phi$, and longitude directions are also plotted. We have also added the Galactic bar at an orientation angle of $14^{\circ}$ (following Freudenreich 1998) with length $3 \mathrm{kpc}$ (following Vallee 2005). Note however that a recent Galactic Legacy Mid-Plane Survey Extraordinaire (GLIMPSE) study by Benjamin et al. (2005) report on a linear Galactic bar with half-length of $4.4 \pm 0.5 \mathrm{kpc}$.

(minimum $\chi^{2}$, as reported in Eq. (1)) is easier to determine, having a relatively small $0.2^{\circ}$ error. The second field, $240^{\circ} \leq$ $l \leq 250^{\circ}$, is less populated and the error is higher reaching $0.5^{\circ}$.

The lower panels of Fig. 4, show an example of deriving the best warp angle for latitude profiles between $220^{\circ} \leq l \leq 230^{\circ}$ from the $R_{\odot}=7.3 \mathrm{kpc}$ RGB sample. The upper panel shows the Northern and Southern latitude profile assuming no warp, i.e. a symmetry axis around $b=0^{\circ}$. The lower panel shows the same two profiles assuming a symmetry axis around $b=-2.9^{\circ}$, as derived when the differences between the folded profiles are minimized and the symmetry angle is allowed to vary within $|b| \leq 5^{\circ}$. One may note that for large warp angles the Southern latitude profile becomes shorter than the Northern one. This is due to the warp angle becoming significant compared to the imposed angular limit in the extraction; i.e. $|b| \leq 20^{\circ}$.

Once the warp angle has been derived for each line of sight we proceed in estimating the vertical density profile of the stellar disk: the scale-height $\left(Z_{\mathrm{h}}\right)$. Our intent is to characterize the radial trend of the stellar disk scale-height. A disk flaring is usually seen as an increasing scale-height towards the outer parts of the disk. An example of the adopted procedure is shown in Fig. 5. Having derived the warp angle, the $b$ angles were transformed in linear $Z$ height according to the distance

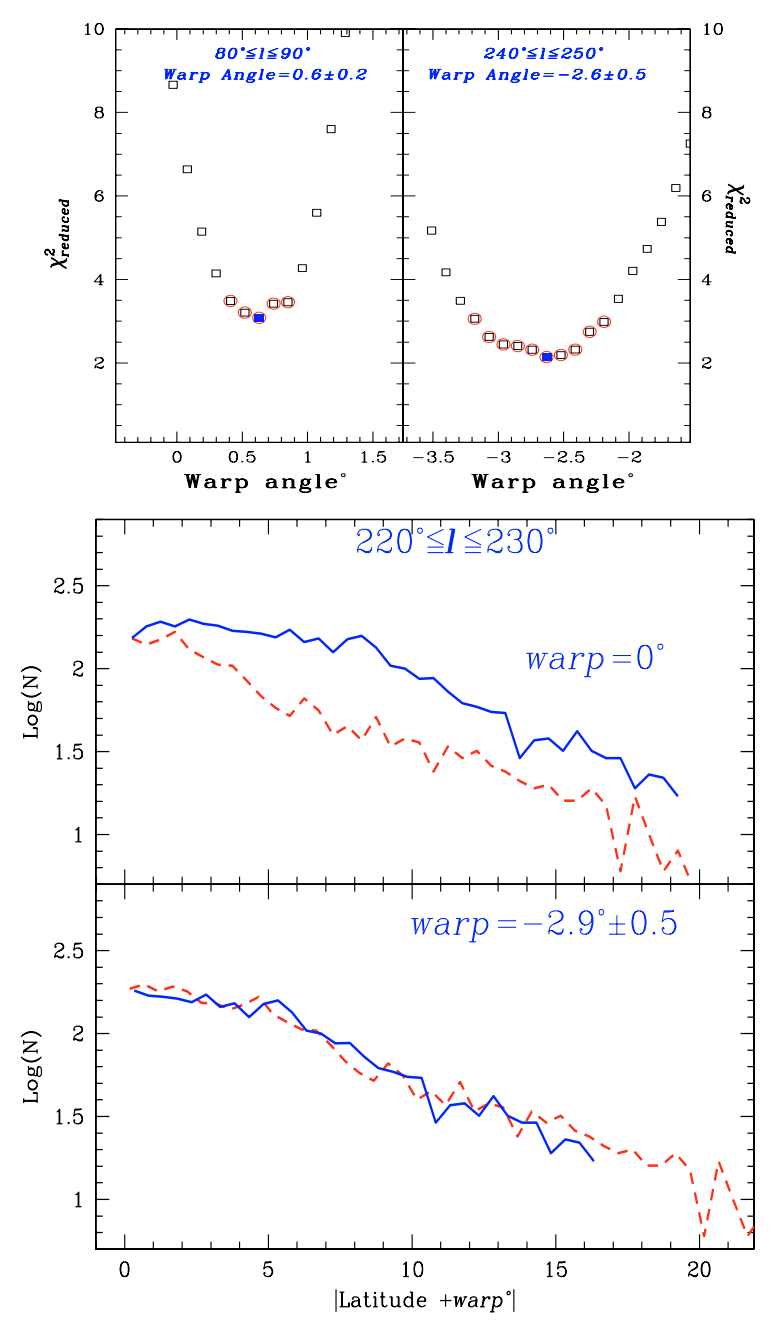

Fig. 4. The upper panels display two examples of the derivation of best warp angles and their relative error by means of a reduced $\chi^{2}$. The lower panels show the latitude profiles for a third field: (i) assuming no warp (upper panel) and; (ii) having derived the best warp angle (lower panel). The dashed and solid lines refer to the Northern and Southern latitude profiles, respectively. All panels refer to the RGB sample at $R_{\odot}=7.3 \mathrm{kpc}$.

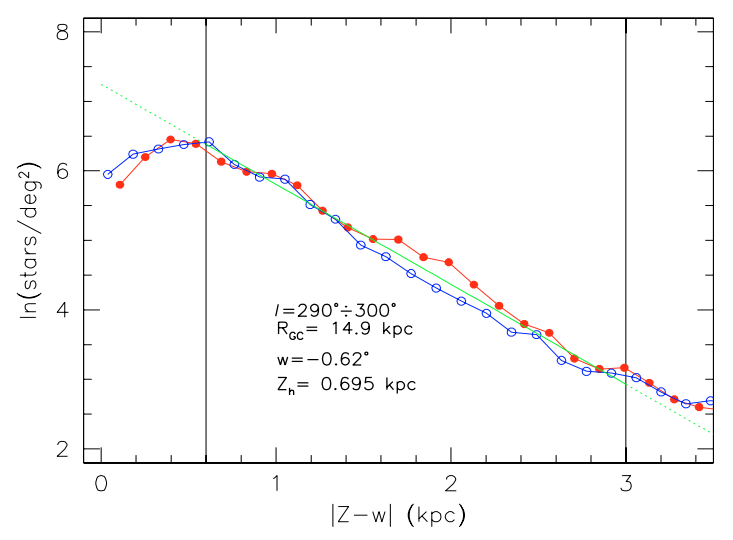

Fig. 5. An example of deriving the scale height $\left(Z_{\mathrm{h}}\right)$ from the RGB sample at $R_{\odot}=16.6 \mathrm{kpc}$. The filled circles show the Northern hemisphere latitude profile while the open circles show the Southern profile. Vertical lines delimit the region where we apply a linear fit (dotted line) to the data. 
from the Sun of the sample. Then the Northern and Southern profiles were overlapped and the star density profile was fitted by a power law, thus deriving $Z_{\mathrm{h}}$. The fitting of the vertical density profile was made within 2 limits in $Z$, so as to exclude: (i) the highly obscured inner regions within $\pm 2.0^{\circ}$ from the mid-plane and; (ii) the very outer regions where in cases of high warp angles the latitude profile of one hemisphere is short with respect to the other hemisphere (cf. the latitude profiles in lower panel of Fig. 4).

The vertical $Z$ scale-height was fitted by a single exponential. We have not tried to use a more complicated formula like the sech or double exponential (see for example the analysis by Alard 2000). Thus, our analysis is aimed at measuring the order of magnitude of the flare in the outer disk. A detailed parametrization of the flare (in terms of thin-thick disk separation), although very interesting, is beyond the scope of this paper. In particular, we note that for the regions of most interest (the outer disk) any thin disk flaring would act in a way that increases the confusion between the thin and thick disk components. This in-ability in separating thin-thick disk components in the outer regions is exacerbated by the unavailability of kinematic all-sky data, which might allow a separation of the two components. Thus, for the outer disk, one is left with determining only an approximate amplitude of any flare. Figure 5 shows an example of the vertical density profile fit for the RGB sample at a heliocentric distance of $16.6 \mathrm{kpc}\left(R_{\mathrm{GC}} \simeq 14.9 \mathrm{kpc}\right)$. The abscissa, $|Z-w|$, indicates the linear $Z$ height folded around the mid-plane of the disk as determined by the warp angle analysis. The analysis of the radial trend of the vertical scale height, $Z_{\mathrm{h}}$, for the RGB sample will be discussed in Sect. 5 .

\subsection{Comparing DIRBE integrated surface photometry with $2 M A S S$ star counts}

In this paper we compare the derived stellar warp with that obtained for other Galactic components, including integrated light, neutral gas and the interstellar dust. In regards to this, we mainly make use of the Freudenreich et al. (1994) study where the warp due to dust and integrated light has been derived using the Diffuse Infrared Background Experiment (DIRBE) mapping of the Galactic plane (see also Vig et al. 2005).

The results of DIRBE however come from integrated light surface photometry which sum contributions from composite stellar populations at different distances and luminosities, and therefore these can be correctly compared with the 2MASS results only after a discussion of their relative weight. The DIRBE $1.2 \mu \mathrm{m}$ and $2.2 \mu \mathrm{m}$ spectral bands correspond roughly to the infrared $J$ and $K$ pass-bands. It is important to note that the DIRBE near-infrared $(\lambda<5 \mu \mathrm{m})$ emission is dominated by stellar disk stars [whereas in far-infrared bands (e.g. $240 \mu \mathrm{m}$ ) the emission by interstellar dust dominates].

To better understand which stellar populations contribute most in the DIRBE emission maps, we used the 2MASS star counts to integrate the light coming from specific stellar populations. We limit this analysis to only one line of sight (the 2MASS color-magnitude diagram presented in the lowest panel of Fig. 2), and expect this to be representative of any other
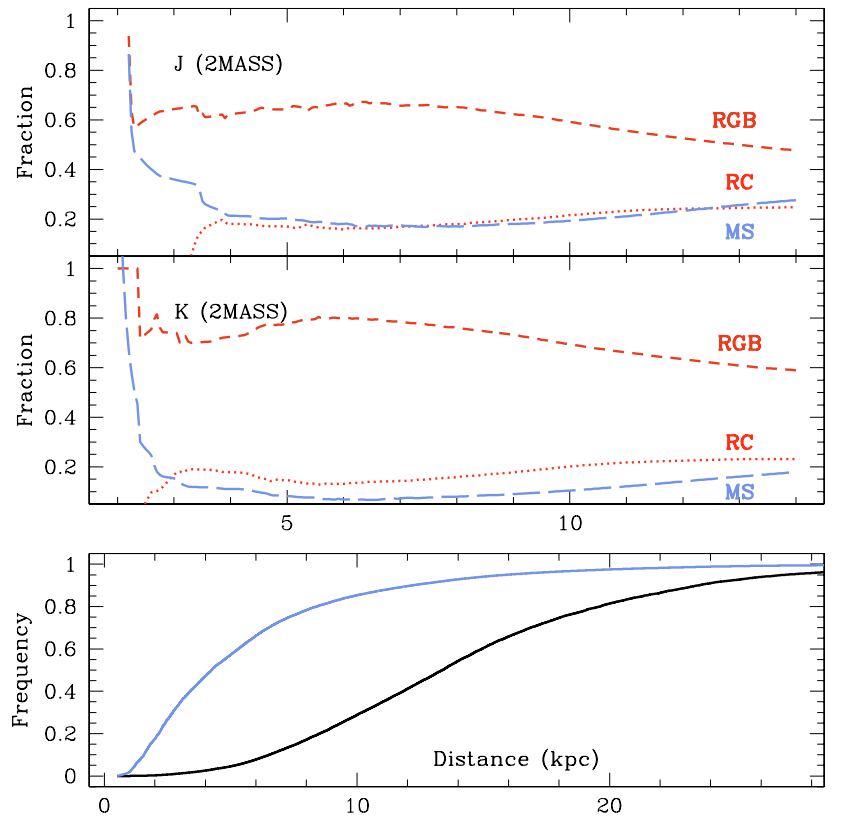

Fig. 6. The relative luminosity contribution of the three stellar populations in the $J$ band (upper panel) and $K$ band (middle panel). The lower figure shows the cumulative distributions of the distances of RGB stars without any luminosity-weighting along the line of sight (dark line). The grey line shows instead the distance-weighted distribution.

line of sight. We roughly disentangle three main populations in this 2MASS color magnitude diagram: (i) MS objects with $(J-K)<0.45$, mainly main sequence and blue super-giants stars; (ii) RC stars with $0.45<(J-K)<0.65$; and (iii) RGB, red super-giant and asymptotic branch stars with $(J-K)>0.65$. For each of these three stellar populations we summed up the light contribution coming from the single point sources (stars) falling within the above color intervals.

In Fig. 6 we show the relative luminosity contribution of the three stellar populations in the $J$ band (upper panel) and $K$ band (middle panel). A limiting magnitude of $K=14.0$ has been applied in estimating the cumulative and relative luminosity of the three different stellar populations. The middle panel, displaying the relative luminosity contribution of the three populations to the total luminosity, shows clearly that in the case of the $K$ band the total light contribution of MS stars is almost negligible in DIRBE, contributing only $15 \%$ of the total light. On the other hand, RGB stars contribute up to $60 \%$ of the total light, a level that goes up to $82 \%$ once also the RC are summed together with RGB stars. Thus we conclude that the level of contamination by MS stars in $2.2 \mu \mathrm{m}(\sim K)$ DIRBE emission maps is fairly low and that red populations dominate the integrated light. Similar conclusions can be drawn for the shortest DIRBE wavelength band at $1.2 \mu \mathrm{m}(\sim J$, upper panel): the RGB and RC populations dominate the DIRBE light, contributing up to $75 \%$ of the total light.

We also checked the weighted distance range at which DIRBE is more sensitive. Considering that the RGB population is the dominant population in both $J$ and $K$ bands we calculated their distance distribution using the Majewski calibration of the Sagittarius red giant branch (see Sect. 2.3). The dark line in the 
lowest plot of Fig. 6 shows the cumulative distributions of the distances of RGB stars without any luminosity weighting along the line of sight. The grey line shows the cumulative distance distribution once we weight the stellar distance with the stars light.

Clearly, the DIRBE integrated light is linearly sensitive to distances within $\sim 9 \mathrm{kpc}$ (at least in this direction $l \sim 240^{\circ}$ ) where the light contribution reaches $80 \%$ of the total. This result, based on RGB stars, should not change considerably once we add the contribution of (i) the RC stars which weight more to larger distances; and (ii) the MS stars which weight more to shorter distances. Thus, being of the same size, the two opposite contributions of the MS and RC stars will to first order cancel out.

In conclusion, with the help of the 2MASS star counts we have identified which stellar populations are the main contributers to the DIRBE luminosity, and at what distances these contribute the most. The results are that the RGB stars are the dominant contributors of the $J$ and $K$ DIRBE integrated light, within $\sim 9 \mathrm{kpc}$. This allows us to perform a fruitful comparison of the DIRBE warp results with those obtained from our 2MASS analysis.

\section{The stellar warp as traced by RC stars}

In this section we present the derived stellar warp as traced by RC stars. We anticipate two obvious points: (i) the warp amplitude depends on the employed wavelength; shorter wavelength investigations probe nearer areas and therefore may underestimate the warp amplitude if it increases with distance; and (ii) the warp amplitude derived depends on the contamination of the employed stellar tracer: inclusion of nearby stars in samples of more distant stars will underestimate the warp amplitude at larger distances. Overall, red clump stars are not the ideal tracers of the warp at larger distances, and we will show this using Figs. 7 and 8.

The upper left panel of Fig. 7 displays the stellar warp as derived using RC stars at $R_{\odot} \leq 2 \mathrm{kpc}$. At first sight, the presence of a global stellar warp can be debatable, as it contrasts with the neat sinusoidal function seen in Djorgovski \& Sosin (1989) for IRAS sources. It remains true however that the Southern stellar warp is distinguishable between $190^{\circ} \div 290^{\circ}$. This brings the Galactic mid-plane $\sim 2^{\circ}$ down at around $l \sim$ $250^{\circ}$, and demonstrates that the stellar warp is detectable already in the solar circle. Problems arise when searching for a global stellar warp in the Northern hemisphere. A sudden drop at around $l \sim 120^{\circ}$ seems to interrupt a global warp signature. To understand the absence of a clear stellar warp in the Northern hemisphere we make a comparison with the latitude of peak brightness as derived in Freudenreich et al. (1994) using DIRBE mapping of the Galactic plane. Overall, the DIRBE data-points at $1.2 \mu \mathrm{m}$ reproduce the global features derived from nearby $R_{\odot} \leq 2 \mathrm{kpc}$ RC stars. In particular both data-sets show an early drop at $l \sim 120^{\circ}$ demonstrating: (i) the consistency of the DIRBE-2MASS comparison; and (ii) the presence of a localized nearby structure. Indeed, the drop in 2MASS and DIRBE traces the presence of the Orion-Cygnus segment (called Local Arm in Russeil 2003).

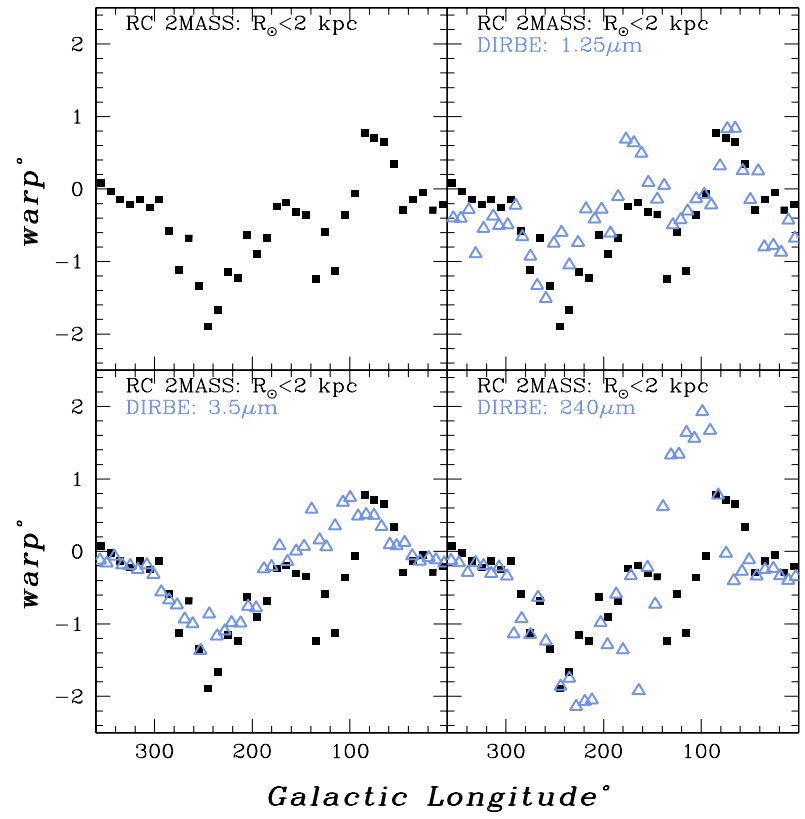

Fig. 7. The stellar warp as derived from RC stars at $R_{\odot} \leq 2 \mathrm{kpc}$ (filled squares). The results are compared with the latitude of peak brightness obtained from DIRBE at wavelengths 1.2, 3.5 and $240 \mu \mathrm{m}$ by Freudenreich et al. (1994, grey open triangles).

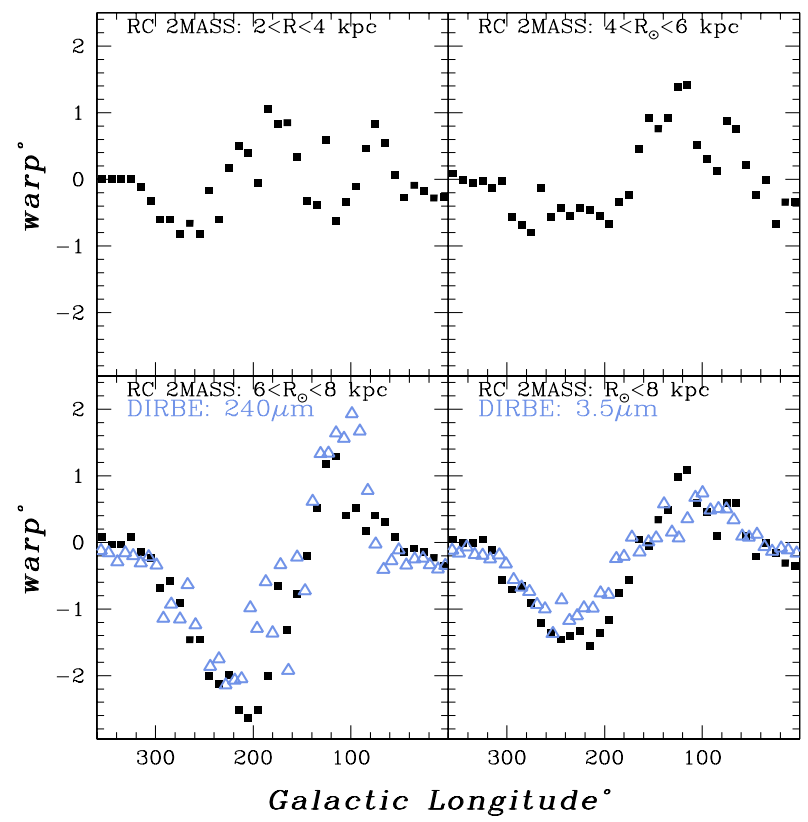

Fig. 8. The stellar warp as derived from RC stars for 4 distance intervals (dark filled squares). The results are compared with the latitude of peak brightness obtained from DIRBE at wavelengths 3.5 and $240 \mu \mathrm{m}$ by Freudenreich et al. (1994, grey open triangles).

The lower left panel of Fig. 7 over-plots the DIRBE datapoints at $3.5 \mu \mathrm{m}$. As anticipated before, at $\lambda<5 \mu \mathrm{m}$ DIRBE emission is still dominated by disk stars. However, as one moves to longer wavelengths DIRBE data-points sample more distant structures that were obscured at shorter wavelengths. This explains why at $3.5 \mu \mathrm{m}$ we do not observe the strong drop at $l \sim 120^{\circ}$ (seen at $1.2 \mu \mathrm{m}$ and in RC stars at $R_{\odot} \leq 2 \mathrm{kpc}$ ) and this is replaced by a global, and smooth warp signature. This 
effect is further demonstrated when comparing the warp obtained for RC stars at $R_{\odot} \leq 2 \mathrm{kpc}$ with that due to the dust (traced at $240 \mu \mathrm{m}$ ). The dust warp does not show the drop at $l \sim 120^{\circ}$ anymore, and although still fluctuating, overall a global and large-scale structure (the warp) is identified. We emphasize however that a simple comparison between the warp of $\mathrm{RC}$ stars at $R_{\odot} \leq 2 \mathrm{kpc}$ with that at $240 \mu \mathrm{m}$ is rather improper, since the DIRBE dust warp is sensitive to more distant regions. The comparison has been meant to show how increasing the adopted wavelength unveils more distant regions and a larger warp amplitude.

Before drawing our conclusions on the RC stars as a stellar warp tracer, we analyze the impact of their contamination status on the global warp properties. This is illustrated in Fig. 8, which displays the stellar warp as derived from RC stars at heliocentric distances between $2 \div 4,4 \div 6,6 \div 8$ and $\leq 8 \mathrm{kpc}$. The upper panels of Fig. 8 prove again how the presence of a global warp can be debatable for the "nearest" RC samples. Things change considerably for RC stars between $6 \leq R_{\odot} \leq 8$. At this distance a global warp signature is evident in both hemispheres. The warp is regular and shows a strong asymmetry in the maximum warp amplitude. At $l \simeq 210^{\circ}$ the warp brings the Galactic mid-plane $\sim 2.7^{\circ}$ below the nominal $b=0^{\circ}$, whereas in the Northern hemisphere the maximum warp amplitude stops at $b \simeq+1.5^{\circ}$. Comparing the warp obtained for the $6 \leq R_{\odot} \leq 8 \mathrm{kpc}$ RC sample with DIRBE data shows excellent agreement with that obtained at $240 \mu \mathrm{m}$ tracing the dust. This indicates that the RC star sample between $6 \leq R_{\odot} \leq 8 \mathrm{kpc}$ (although it includes up to $30 \%$ contamination by local dwarfs) still enables sufficient isolation of distant stars, those whose warp signature matches that of DIRBE at $240 \mu \mathrm{m}$.

The impact of contamination on the retrieved warp amplitude is best illustrated in the lower right panel of Fig. 8. With respect to the warp derived from RC stars between $6 \leq R_{\odot} \leq$ $8 \mathrm{kpc}$, those between $0 \leq R_{\odot} \leq 8 \mathrm{kpc}$ show a significant change in the amplitude of the warp maximum and location. In particular, the maximum warp amplitude passes from $\sim-2.7^{\circ}$ (for $\mathrm{RC}$ between $6 \leq R_{\odot} \leq 8 \mathrm{kpc}$ ) to $\sim-1.5^{\circ}$ (for $\mathrm{RC}$ at $0 \leq R_{\odot} \leq 8 \mathrm{kpc}$ ). A comparison with DIRBE data now shows a better agreement with the $3.5 \mu \mathrm{m}$ data and this is again the expected result. Indeed, both the 2 MASS $0 \leq R_{\odot} \leq 8 \mathrm{kpc}$ star counts and the DIRBE $3.5 \mu \mathrm{m}$ surface photometry are sensitive all along the line of sight up to $\sim 8 \mathrm{kpc}$.

In conclusion, the plots presented in Figs. 7, 8 highlight the fact that a thorough characterization of the Galactic stellar warp, in particular its maximum amplitude and maximum location, sensitively depend on the distance and contaminationstatus of the employed tracer. The lower panels of Fig. 8 are the most significant in demonstrating how the maximum stellar warp can shift its location by $\sim 40^{\circ}$ in longitude and can halve its amplitude depending on the contamination degree of the adopted tracer. In addition, the lower right panel of Fig. 8 proves the validity of our method in tracing the stellar warp, and the consistency of the comparison between DIRBE and 2 MASS.

This being established we will now focus our discussion on the stellar warp as derived using red giants. As anticipated in Sect. 2.3, the red giant samples include less contamination by local dwarfs and enable a better and more reliable separation of more distant stars.

\section{The stellar warp as traced by RGB stars}

Figures 9 and 10 display the stellar warp as derived from RGB samples at $R_{\odot}=7.3$ and $R_{\odot}=16.6 \mathrm{kpc}$. A global regular warp signature is clearly evident in both samples, reflecting large-scale Galactic structure (see Sect. 4.2). The most striking feature for the RGB $R_{\odot}=7.3 \mathrm{kpc}$ sample is the amplitude of the Southern maximum warp, which lowers the Galactic mid-plane by almost $3^{\circ}$. By applying a Gaussian fit to the data-points between $160^{\circ} \leq l \leq 310^{\circ}$, we estimate a mean warp maximum at $l \sim 235^{\circ}$. Similarly, for the $R_{\odot}=16.6 \mathrm{kpc}$ RGB sample the warp maximum is estimated at $l \sim 240^{\circ}$.

The lower left panel of Fig. 9 shows a comparison between the stellar warp as derived from the RC star samples ( $6 \leq R_{\odot} \leq 8$ and $0 \leq R_{\odot} \leq 8 \mathrm{kpc}$ ) with the RGB sample at $R_{\odot}=7.3 \mathrm{kpc}$. This comparison is an excellent demonstration of how (i) the distance and (ii) the contamination status of the adopted stellar tracer affect the discussion on the location and amplitude of the maximum stellar warp. The RC sample between $6 \leq R_{\odot} \leq 8 \mathrm{kpc}$ shows a comparable Southern warp maximum amplitude with the RGB sample at $R_{\odot}=7.3 \mathrm{kpc}$, and this reflects the fact that both samples refer to the same distance range. Yet, the location of the warp maximum for the RC sample between $6 \leq R_{\odot} \leq 8 \mathrm{kpc}$ is $\sim 20^{\circ}$ shifted with respect to that for the RGB sample at $R_{\odot}=7.3 \mathrm{kpc}$. On the other hand, the more contaminated $\mathrm{RC}$ sample between $0 \leq R_{\odot} \leq 8 \mathrm{kpc}$ shows a better agreement with the RGB sample at $R_{\odot}=7.3 \mathrm{kpc}$ in the mean location of the warp maximum around $l \sim 240^{\circ}$. However, the maximum amplitude of the RC sample between $0 \leq R_{\odot} \leq 8 \mathrm{kpc}$ has clearly decreased, a reminder of how the inclusion of nearby stars leads to an under-estimated warp amplitude.

We now turn our attention to the comparison of the stellar warp (derived from the RGB sample at $R_{\odot}=7.3 \mathrm{kpc}$ ) with that obtained for the interstellar dust and neutral atomic hydrogen components. The upper right panel shows the comparison with the DIRBE data at $240 \mu \mathrm{m}$. The agreement between the stellar and dust warp is evident. The two share the same warp phase-angle and show only small differences in the Southern maximum amplitude.

A recent upgrade of the Drimmel \& Spergel (2001) Galactic dust model, using DIRBE $240 \mu \mathrm{m}$ data, shows the presence of an extended spiral arm entering the third quadrant and intersecting the suggested location of CMa. The reconstruction of the Galactic dust distribution is always model dependent. However, the new model (Drimmel 2005) indicates that the far infrared DIRBE $240 \mu \mathrm{m}$ data, are sensitive to at least $\sim 7 \mathrm{kpc}$ from the Sun, and possibly beyond. The distance consistency of the far infrared DIRBE $240 \mu \mathrm{m}$ data with the RGB star sample at $R_{\odot}=7.3 \mathrm{kpc}$ (and given the excellent agreement presented in the upper right panel of Fig. 9) allows us to ascertain that the dust and stars at CMa distances are similarly warped.

Besides the excellent agreement between the dust and stellar warp, a similar conclusion is also obtained for the gaseous 

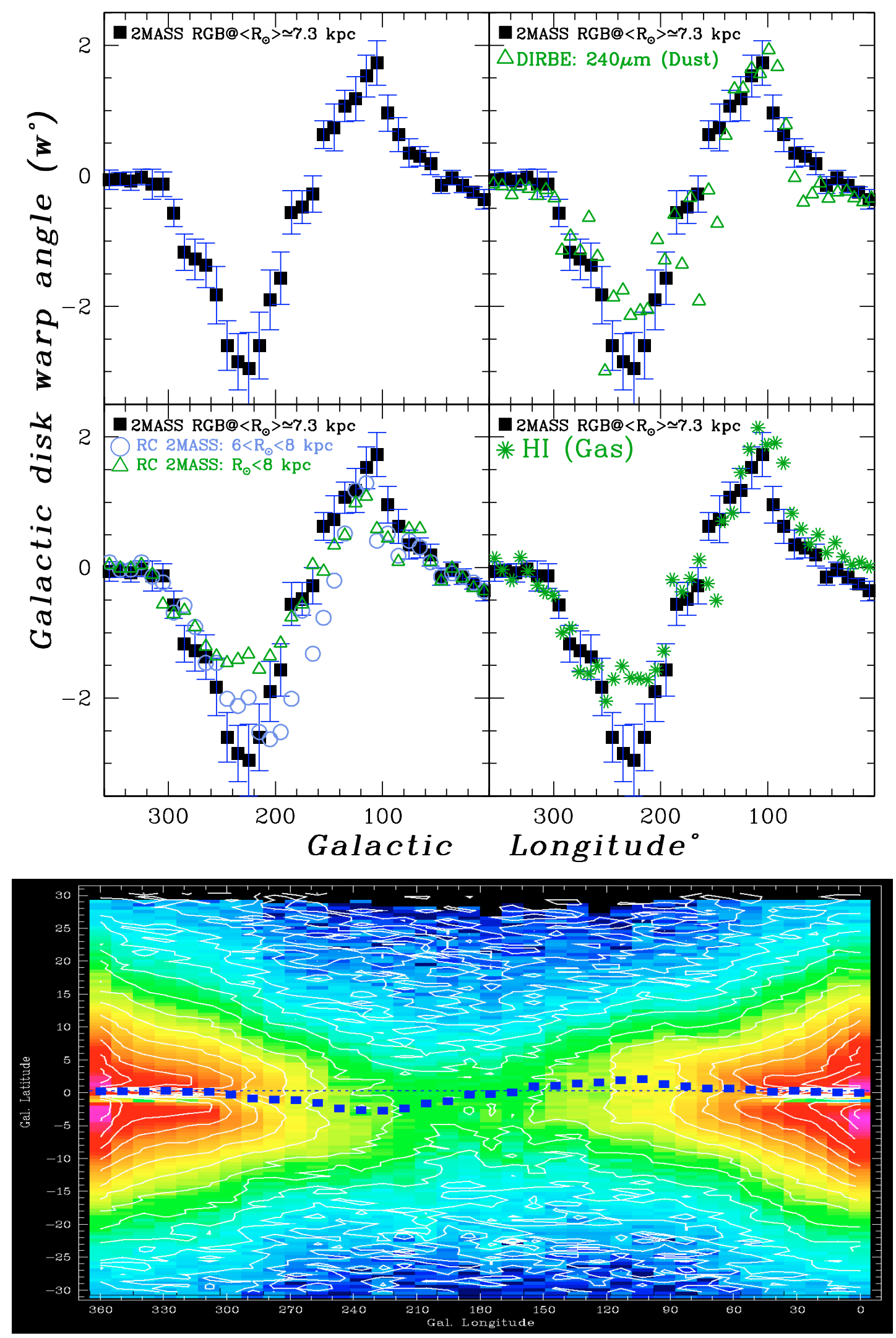

Fig. 9. The upper left panel displays the stellar warp as derived from red giants at $R_{\odot} \simeq 7.3 \mathrm{kpc}$. In the lower left panel we compare the derived warp with those based on two RC samples between: (i) $6 \leq R_{\odot} \leq 8$, and (ii) $0 \leq R_{\odot} \leq 8 \mathrm{kpc}$. The upper right panel over-plots the dust warp as derived from DIRBE at $240 \mu \mathrm{m}$ data, whereas the lower right panel over-plots the Galactic warp as derived from neutral H I gas (also from Freudenreich et al. 1994). The lower panel shows the density maps and contours of the $R_{\odot} \simeq 7.3$ RGB sample. One can trace by eye the warp signature (a colored version is more appropriate). Over-plotted is also the location of the mean mid-plane warped disk as a function of longitude. 


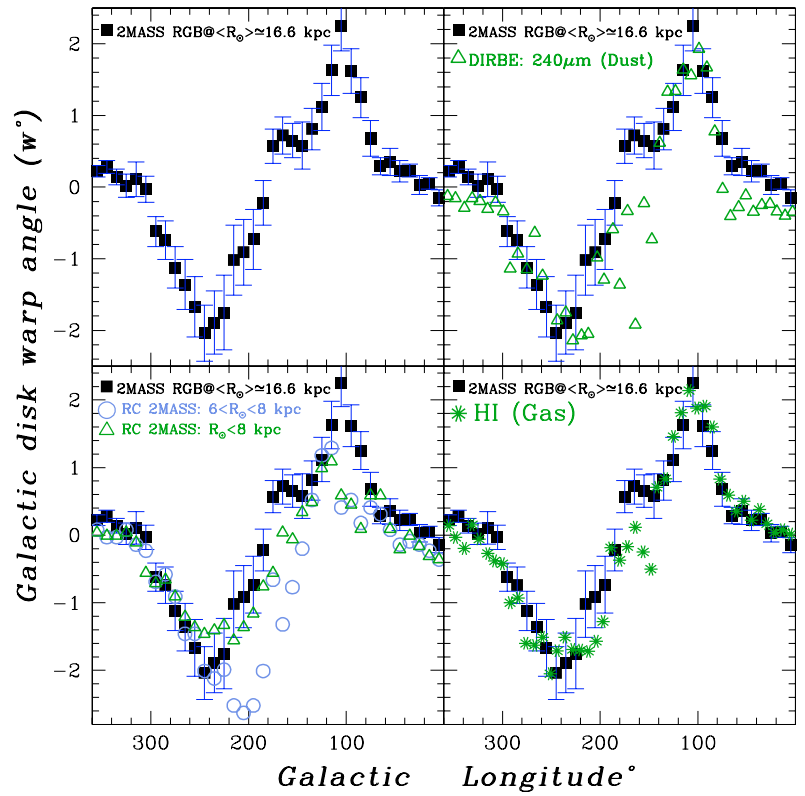

Fig. 10. Same as Fig. 9, but for RGB stars at $R_{\odot} \simeq 16.6 \mathrm{kpc}$. Note the structure ("swelling") around $l \sim 160^{\circ}$ and how the the agreement with $\mathrm{H}$ I is better with respect to the $R_{\odot} \simeq 7.3 \mathrm{kpc}$ sample.

and stellar warp. The Hi latitude of peak brightness as derived by Freudenreich et al. $\left(1994^{3}\right)$ follows the same warp signature we have obtained from the RGB sample at CMa distance. The only obvious difference is related to the maximum Southern warp amplitude of H I that seems to saturate; showing an almost constant $w$ warp-angle of $-1.75^{\circ}$ for longitudes between $210^{\circ}$ and $270^{\circ}$. Might this observed difference between the amplitude of the stellar and gaseous warp suggest an "accretion-perturbation" on top of the large-scale warp structure derived in this paper?. In regard to the reality of this effect, one must keep in mind that over the last few years there has been alternating evidence on warp amplitude differences between stars and gas. Indeed, we remind the reader that the disk radial truncation hypothesis at $R_{\mathrm{GC}} \simeq 14 \mathrm{kpc}$ was first proposed (Freudenreich et al. 1994; Porcel et al. 1997) in order to explain "why the stellar warp seemed half the amplitude of the gaseous warp". Thus, our results which go in the opposite direction (stellar RGB warp at $R_{\odot} \simeq 7.3 \mathrm{kpc}$ showing twice the H I warp amplitude) should only be read in terms of the different confusion status, and therefore of the mean probed distances, of the gas.

Indeed, whereas we are able to isolate the warp signature due only to the stellar populations at $R_{\odot} \simeq 7.3 \mathrm{kpc}$, the gaseous warp derived by Freudenreich et al. (1994) is that due to the summing of different warp signatures by gas distributed along the line of sight, at different distances. This is a direct consequence of the inability to recover gas distribution distances in lines of sight without clear kinematical signatures. Thus the saturation effect seen in the H I warp may reflect that seen for

${ }^{3}$ Note that although the Freudenreich et al. paper was based on DIRBE data, they also derived the gaseous warp based on a reanalysis of the velocity-integrated HI emission maps from the survey of Weaver \& Williams (1973). the stellar warp (cf. the lower panels in Fig. 8 and the lower left panel in Fig. 9) when including nearby stars in distant star samples. More fundamentally, leaving apart the flattening of the gas warp in the range $210^{\circ} \leq l \leq 250^{\circ}$, one notes excellent agreement between of the gas and stellar warp profiles at all the other viewing angles.

Moving to the stellar warp derived by RGB stars at $R_{\odot} \simeq$ $16.6 \mathrm{kpc}$ (Fig. 12) one notes structure ("swelling") between $140^{\circ} \leq l \leq 180^{\circ}$. Is this a deviation from a smooth global warp signature?. We suggest that this is the same effect seen for nearby RC samples, and it is due to the Orion-Cygnus Local Arm (short dashed line in Fig. 3). This "swelling" is not seen in the $R_{\odot} \simeq 7.3 \mathrm{kpc}$ sample because the selected RGB stars are bright enough to avoid the faintest regions of the diagrams (mostly populated by nearby dwarfs). On the contrary, the RGB sample at $R_{\odot} \simeq 16.6 \mathrm{kpc}$ can be easily contaminated by nearby $R_{\odot} \leq 2 \mathrm{kpc}$ dwarf stars (compare the upper and lower panels of Fig. 2) which would enter our selection box if subject to high reddening or photometric error. In turn, the structure appears in the $R_{\odot} \simeq 2.8 \mathrm{kpc}$ RGB sample (Fig. 12) supporting our interpretation that the "swelling" is due to nearby dwarf stars that contaminate primarily the $R_{\odot} \simeq 16.6 \mathrm{kpc}$ RGB sample. Given the similarities between the $R_{\odot} \simeq 2.8$ and $16.6 \mathrm{kpc}$ warps, to what extent might the $R_{\odot} \simeq 16.6 \mathrm{kpc}$ warp signature be in fact a signature of nearby contamination in the $R_{\odot} \simeq 16.6 \mathrm{kpc}$ RGB sample? The amplitude difference in the Northern warp of the $R_{\odot} \simeq 2.8$ and $16.6 \mathrm{kpc}$ samples however argues against this possibility (compare Figs. 12 and 13).

Recently McClure-Griffiths et al. (2004) presented an H I study from the Southern Galactic Plane Survey (SGPS), and pointed to the possible presence of a distant spiral arm in the fourth quadrant of the Milky Way. The distinct and cohesive feature has been traced over $70^{\circ}$ and is located between $18 \leq R_{\mathrm{GC}} \leq 24 \mathrm{kpc}$. In regards to this, it is interesting to note that our detection of a stellar warp in the RGB sample at $R_{\odot} \simeq 16.6 \mathrm{kpc}$ might represent the stellar counter-part of this distant H I spiral arm. Indeed, the RGB sample at a mean distance of $R_{\odot} \simeq 16.6 \mathrm{kpc}$ probes the stellar populations between $13.2 \div 20.0 \mathrm{kpc}$ from the Sun (Fig. 3) and this is compatible with the mean position of this $\mathrm{HI}$ arm being within $\sim 20 \mathrm{kpc}$ from the Galactic center (cf. Fig. 3a of McClure-Griffiths et al. 2004).

Interestingly, McClure-Griffiths et al. find that this distant arm is well-confined to the Galactic plane, dropping at most by $\sim 1 \mathrm{kpc}$ below the Galactic equator. This again is in agreement with our result (Fig. 13) that the Galactic mid-plane (at these distances and lines of sight) is located at $\sim 0.5 \mathrm{kpc}$ below latitudes of $b=0^{\circ}$. The SGPS H I survey analysis however remains limited to $l \sim 250^{\circ}$ (McClure-Griffiths et al. 2005), so that a detailed comparison of the stellar-gaseous warp and the interpretation of this part of the outer disk awaits the completion of the third quadrant. In conclusion, and as we shall argue in Sect. 5, the detection of the warp in the $R_{\odot} \simeq 16.6 \mathrm{kpc}$ sample proves that the MW stellar disk is not truncated at $R_{\mathrm{GC}} \simeq 14 \mathrm{kpc}$, and that a more extended stellar component is present. 


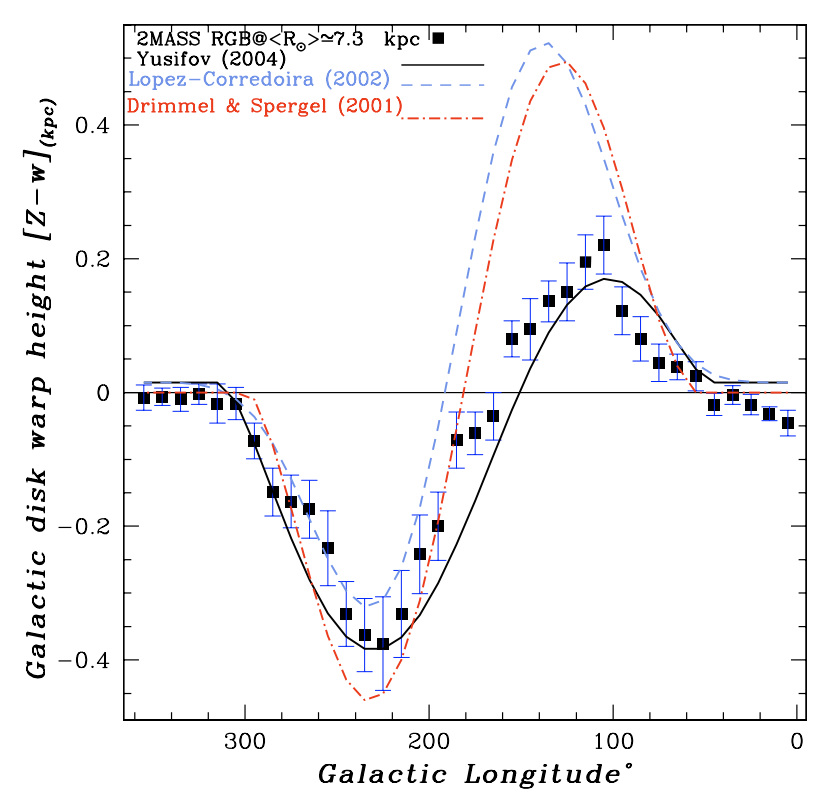

Fig. 11. A comparison of our Galactic stellar disk warp height $Z-w a r p$ (kpc), as obtained from the RGB sample at heliocentric distances of $7.3 \mathrm{kpc}$ points with error bars, with models based on stellar tracers lines. Note the excellent agreement between data and models for the Southern warp. The disagreement for the Northern warp is explained in the text.

\subsection{Comparison with warp models}

Before discussing the possible implications of the warp as determined empirically here on the CMa over-density, we first compare our results with other available models. In Fig. 11 we show a comparison of our derived $Z$ - warp at the proposed distance of $\mathrm{CMa}\left(R_{\odot} \simeq 7.3 \mathrm{kpc}\right)$ with available models based on stellar tracers, namely Drimmel \& Spergel $(2001)^{4}$, López-Corredoira et al. (2002) and Yusifov (2004). The 3 models used the distribution of integrated star light, red clump and pulsar stars, respectively. In the following all models were converted to $R_{\odot}=8.5 \mathrm{kpc}$. At first sight, one sees how all three models converge on showing a warp maximum near $l=240^{\circ}$ rather than $l=270^{\circ}$, a clear hint of the association of CMa with the warp maximum amplitude. Most importantly, at a heliocentric distance of $7.3 \mathrm{kpc}$ the 3 models show excellent agreement with our observed warp amplitude around $l \sim 240^{\circ}$.

One also notes that there exists: (i) an overall good agreement between the López-Corredoira et al. (2002) and Drimmel \& Spergel (2001) models; (ii) a significant dis-agreement between these two models and the Yusifov (2004) model; and (iii) significant agreement between our observed stellar warp and the Yusifov (2004) model. In Sect. 2.1 we showed that the Yusifov (2004) model is based on a rather incomplete catalog, thus the closer agreement of our retrieved warp with this model was an un-expected result. To solve this confusion however, one must go into the details of the models.

The 3 models describe the warp as a series of concentric rings which intersect the Galactic plane along a line of

\footnotetext{
${ }^{4}$ Note that in this paper we do not use the Drimmel \& Spergel (2001) model that is based on dust, since this predicts very high warping (cf. Fig. 2 of Yusifov 2004).
}

nodes (usually measured as the Galactocentric angle from the sun to the Galactic center $\phi$, see Fig. 3 and Fig. 1A of Evans et al. 1998). The models of López-Corredoira et al. (2002) and Drimmel \& Spergel (2001) report $\phi=-5^{\circ}$ and $0^{\circ}$ respectively. On the other hand, the Yusifov (2004) model derives a rather higher $\phi=+15^{\circ}$. $\phi$ different than zero means that the Sun is not located on the line of nodes, instead it is already inside one of the two oppositely warped regions. As a consequence, when viewed from the Sun, geometrically the warp maximum is nearer to us in one hemisphere than it is in the other. Thus, if the warp is traced at fixed distances from the Sun and $\phi \neq 0$, then the observed warp is asymmetric. Yusifov (2004) derives a positive $\phi$ value and therefore predicts a significantly asymmetric warp, with a relatively stronger Southern warp maximum amplitude. On the contrary, López-Corredoira et al. (2002) derive a negative $\phi$ value which produces an observable asymmetric warp, with a relatively stronger Northern warp maximum amplitude. Lastly, Drimmel \& Spergel (2001) derive $\phi=0^{\circ}$ and therefore their model predicts a symmetric warp; equivalent Northern/Southern warp maximum amplitudes.

Another factor that contributes to the appearance of an asymmetric warp is due to the chance location of the Northern warp just behind the Norma-Cygnus arm (labeled outer in Fig. 3). Thus, extinction in the Norma-Cygnus arm and possible variations of the extinction curve (due to the penetration of gaseous or dusty regions), coupled with the possibility that the Sun may not lie on the line of nodes are all factors that conspire in producing a less pronounced apparent Northern warp at heliocentric distances of 2.8 and $7.3 \mathrm{kpc}$.

To develop further the particular issue of a symmetric and asymmetric stellar warp, we now compare the stellar warp as traced by the 3 RGB samples at different heliocentric distances. Since our results favor rather high and positive $\phi$ values we will continue the comparison with the Yusifov model as this has shown the best fit to our RGB sample at heliocentric distance $7.3 \mathrm{kpc}$. Figure 12 shows clearly that the Yusifov model provides a satisfactory match with the stellar warp as derived by the $R_{\odot} \sim 2.8$ and $\sim 7.3 \mathrm{kpc}$ RGB samples. Figure 12 also shows how the Northern warp amplitude is almost half that of the Southern warp, for the two distances.

In principle, the warp derived at $R_{\odot} \sim 16.6 \mathrm{kpc}$ should not be compared with the Yusifov model (Fig. 13) since that model is not applicable at $R_{\mathrm{GC}} \geq 14 \mathrm{kpc}$. Nevertheless, for the derived warp at $R_{\odot} \sim 16.6 \mathrm{kpc}$ we find that the Yusifov model predicts maximum warp amplitudes that are not very far from our derived values; with amplitude differences of 0.25 and $0.15 \mathrm{kpc}$ above and below the mid-plane, respectively. Moreover, the location of the warp maxima are still close to those of the derived data-points, indicating that the warp phase angle is still in agreement with observations. Figure 13, also shows another important finding: only at distances of $R_{\odot} \simeq 16.6 \mathrm{kpc}$ can we obtain a good symmetry between Northern and Southern warp maximum amplitudes; both being around $\sim 0.6 \mathrm{kpc}$ above and below the mid-plane. The fact that we obtain a symmetric warp at $R_{\odot} \sim 16.6 \mathrm{kpc}$ also indicates that probing the warp at such large distances is affected less by the dependence on $\phi$.

The passage from the stellar warp at $R_{\odot} \sim 7.3 \mathrm{kpc}$ to that at $16.6 \mathrm{kpc}$ shows an out-break in the Northern warp 


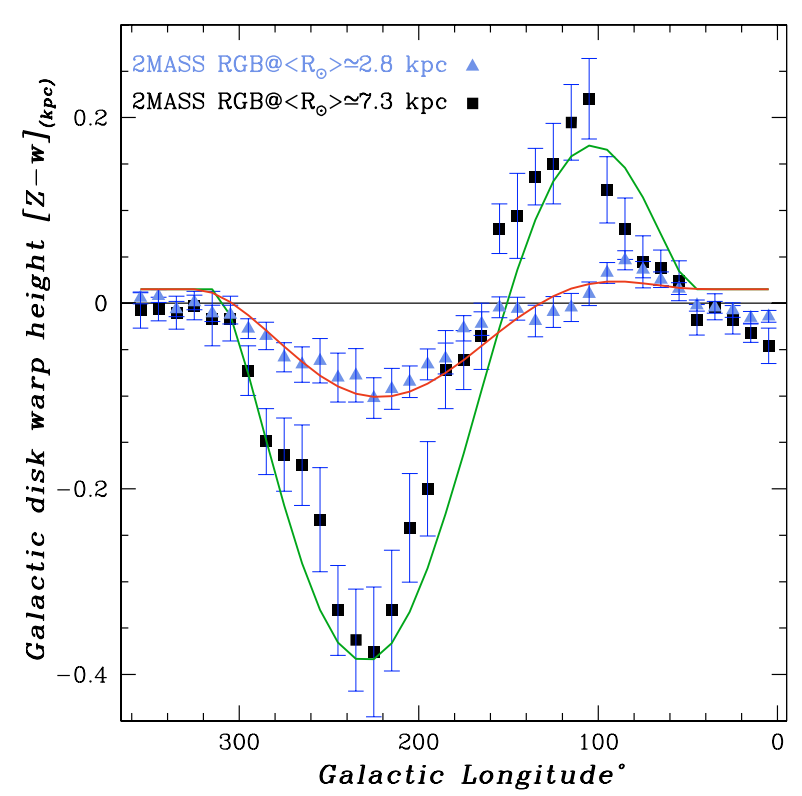

Fig. 12. The Galactic stellar disk warp height as derived from the RGB samples at $R_{\odot}=2.8$ and $7.3 \mathrm{kpc}$ compared with the Yusifov (2004) model.

maximum by almost $0.45 \mathrm{kpc}$. This confirms how probing distant regions (which are less affected by grand-design structures like the Norma-Cygnus arm) reveals the real entity of the Northern warp amplitude. On the other hand, the passage to the stellar warp at $R_{\odot} \sim 16.6 \mathrm{kpc}$ shows a limited increase in the Southern warp maximum amplitude by $\sim 0.2 \mathrm{kpc}$. This limited increase probably reflects the way the Southern gaseous warp shows a saturation effect at $\sim 0.75 \mathrm{kpc}$ below the plane, that induced previous investigations to consider it constant after $R_{\mathrm{GC}}=14.0 \mathrm{kpc}$ (Burton 1998).

\subsection{Significance of the retrieved stellar warp}

Figures 9-13 allow us to conclude the following:

- The correspondance of the CMa over-density with the Southern warp maximum at $l \sim 240^{\circ}$ : this conclusion is based on both our derivation of the stellar warp (at different distances) and independently derived models. This establishes that the warp maximum is significantly displaced from $l \simeq 270^{\circ}$ at CMa distances.

Now that our results indicate that the Galactic warp at $R_{\odot}=$ $7.3 \mathrm{kpc}$ can bring the mid-plane $\sim 3^{\circ}$ below the nominal $b=0^{\circ}$, star-count comparisons above and below the plane should be made as follows: in the Canis Major direction $\left(l=240^{\circ}\right)$ star counts at CMa core $\left(b \simeq-8^{\circ}\right)$ should be compared with those at $b \simeq+2^{\circ}$. This explains the consistent detection of a CMa overdensity or peculiar signature when comparison fields are taken at $b \simeq+8^{\circ}$ (Martin et al. 2005; Bellazzini et al. 2004). One further piece of evidence which indicates that the CMa detection is the recovery of the Galactic warp Southern hemisphere maximum is found in the so-called structure A. Along with the CMa over-density, structure $A$ is the second over-density found in the Martin et al. (2004a) analysis. Its location (in the Northern

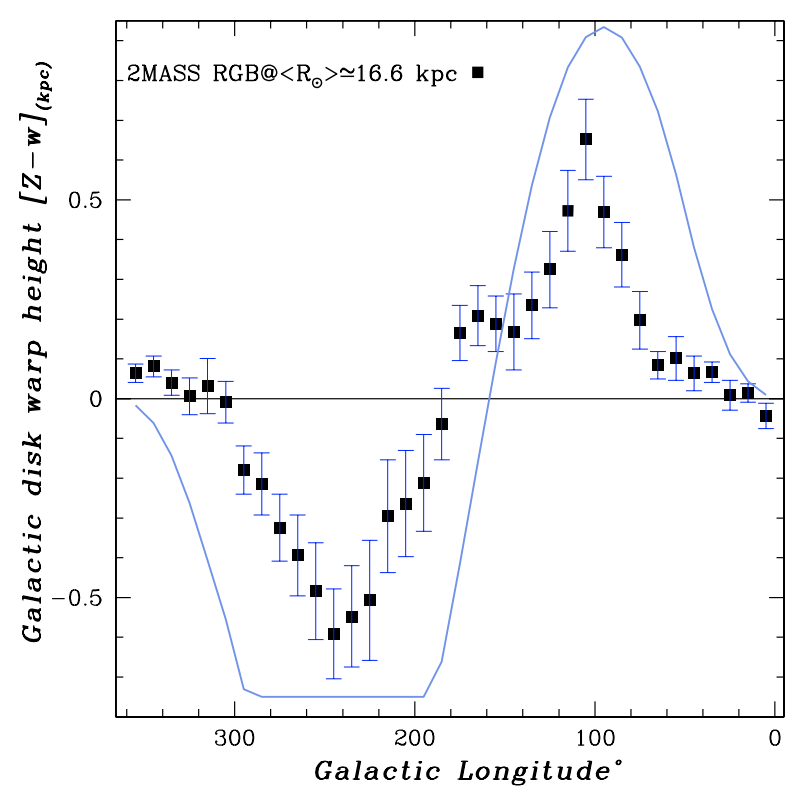

Fig. 13. The Galactic stellar disk warp height as derived from the RGB sample at $R_{\odot}=16.6 \mathrm{kpc}$ improperly compared with the Yusifov (2004) model (not applicable at these distances). Note that for Galactocentric distances greater than $14 \mathrm{kpc}$ the model imposes a constant Southern stellar warp so as to be consistent with the derived parameters of the gaseous warp.

hemisphere) makes it fully compatible with being the Northern warp maximum, as also found in this paper.

Yet another overdensity (in Argo) has recently been announced (Rocha-Pinto et al. 2005). Our results suggest that this over-density cannot be directly correlated to the Galactic warp maximum (as suggested in Bellazzini et al. 2006). Indeed, at $l \simeq 290^{\circ}$ ( $\sim$ Argo center $)$ the Galactic warp amplitude is less pronounced than that at $l \simeq 240^{\circ}$. To consider a possible origin of this over-density, we briefly reconsider the analysis of Rocha-Pinto et al. (2005). In order to increase their accessibility to low latitude sky areas and reduce the dependency on foreground reddening Rocha-Pinto et al. (2005) use a Galactic model to remove the foreground density. The model is idealised in that it assumes a cylindrically symmetric density distribution about the Galactic center. Figure 9 shows that the Galactic warp amplitude at $l \simeq 290^{\circ}$ is not zero, inconsistent with the assumptions of the Rocha-Pinto Galactic model. Although this effect remains to be quantified, and although the warp amplitude is not extreme at $l \sim 290^{\circ}$, the high star density this close to the Galactic disk will amplify the residual Galactic component when Galactic populations are removed, necessarily affecting the quantification of the Argo over-density.

- The regularity of the Galactic stellar warp out to $R_{\mathrm{GC}} \sim$ $20 \mathrm{kpc}$ : We have measured the stellar warp at 3 different distances and argued that the only visible perturbation away from regularity (a "swelling" in the $R_{\odot}=2.8$ and $16.6 \mathrm{kpc}$ rings) can be explained as being due to the presence of the OrionCygnus arm in lines of sight towards the Northern warp and between $140^{\circ} \leq l \leq 180^{\circ}$. That is, we conclude that a global and regular warp signature is traced to Galactocentric distances of at least $20 \mathrm{kpc}$ in the anti-center direction. We emphasise this 
finding, since it is a clear conclusion even though there are two factors that could have masked such a signature: 1) the Galactic flare (see next section) and 2) the decreasing stellar density in the Galaxy's outskirts.

- The consistency of the stellar warp with the dust and gaseous warp: In addition to being a regular large-scale structure, the stellar warp is consistent with that of the interstellar dust and neutral atomic hydrogen. The consistency might have been expected given the close physical correlation between these 3 components. However, it is when considering accretion scenarios that this finding acquires more significance. Accretion of companions can be responsible for generating a short-lived warp, and can modify differentially the stars-dustgas warp properties (cf. Binney 1992). In regards to this, it is interesting to recall that the numerical simulations by Helmi et al. (2003), cited to explain the almost circular orbits of CMa and Mon. Ring, suggest that the accretion event must be relatively young ( $\leq 1 \mathrm{Gyr}$ ) otherwise any coherent structure would be dissolved. Developing consistency between dynamically young events - a few rotation periods - and global regularity remains to be investigated in detail.

- The orientation of the Galactic bar and warp: The results presented in this paper define a stellar warp with a rather high and positive phase-angle. Interestingly, we note that a positive warp phase-angle follows the orientation of the Galactic bar. The amplitude of the warp phase-angle $\left(+15^{\circ}\right)$ in Yusifov's model is very similar to recent determinations of the Galactic bar orientation: Freudenreich (1998) derive $+14^{\circ}$, while Babusiaux \& Gilmore (2005) derive $+22^{\circ} \pm 5.5^{\circ}$. We have shown that the pulsar catalog used by Yusifov is biased towards the Galactic inner regions $\left(R_{\mathrm{GC}} \leq 8 \mathrm{kpc}\right.$, see Fig. 1$)$. Whereas this could have been a shortcoming in his model, it turns out that this inner regions weighted catalog may have led to a better determination of an important warp parameter; its phase-angle at least in the inner Galaxy. Thus, it may be that the Milky Way bar and the inner warp-rings share the same orientation angle. This point will be further investigated in Momany et al. (in prep.). For the time being, we note that our determination of a non-zero phase angle frees us from the uncomfortable assumption of a fortuitous location of the Sun along the line of nodes.

\section{The Galactic flare}

In the present section, and before extending our discussion on the Mon. Ring, we derive another necessary Galactic ingredient, flaring of the Galactic disk.

For this analysis we use the $Z_{\mathrm{h}}$ values of the scale height as determined in Sect. 2.3 for the $3 \mathrm{M}$-giants samples at $R_{\odot}=2.8$, 7.3 and $16.6 \mathrm{kpc}$. The flaring of the Galactic disk (viewed as an increase of the scale-height with increasing Galactocentric distance) is evident in Fig. 14, where we trace the radial trend of $Z_{\mathrm{h}}$. Before discussing the details of Fig. 14, we remind the reader that: (i) we used a single exponential in fitting the vertical density profile, defining a scale-height; (ii) in the fitting process we excluded regions at low latitudes so as to avoid regions of high reddening corrections around the Galactic midplane; and most importantly (iii) our three 2MASS M-giants

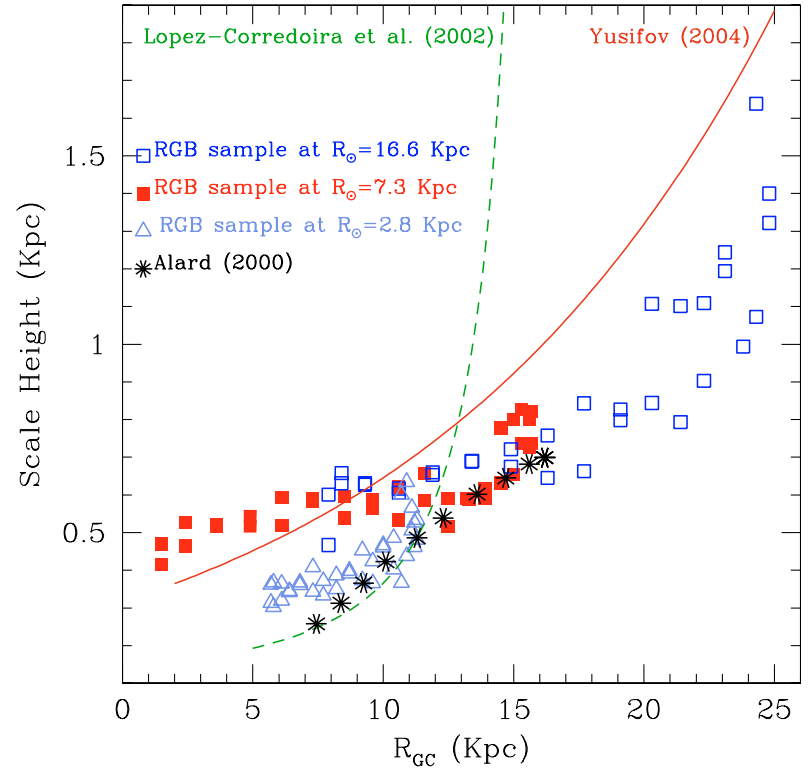

Fig. 14. Variation of the scale-height (derived from the $R_{\odot}=2.8,7.3$ and $16.6 \mathrm{kpc}$ RGB samples) as a function of Galactocentric distances. We also show a comparison of our results with two models and datapoints from Alard (2000).

samples have an upper vertical limit in latitude of $b=+/-20^{\circ}$, and this corresponds to different upper $Z$ probing once the distances of the 3 samples is accounted for. This also means that we have a different weighting of the thin and thick disk stellar populations for the three M-giant samples. Table 1 reports the adopted limits in the vertical density profile fitting, for the three samples. Considering that at the solar distance the scaleheight of the thin disk is $\sim 0.3 \mathrm{kpc}$ while that of the thick disk is $\sim 1 \mathrm{kpc}$ (Wyse \& Gilmore 2005), it is clear that the sample at $R_{\odot} \simeq 2.8 \mathrm{kpc}$ is weighted more by thin disk populations. On the contrary the $R_{\odot} \simeq 7.3$ and $16.6 \mathrm{kpc}$ are weighted more by thick disk populations.

Thanks to the use of different lines of sight at fixed heliocentric distances, our data-points cover a wide range of Galactocentric distances and thus are reliable in tracing the flare all over the Galactic stellar disk (inner and outer). In particular we note the following:

- For Galactocentric distances in the range $4 \leq R_{\mathrm{GC}} \leq$ $15 \mathrm{kpc}$, the $R_{\odot}=7.3 \mathrm{kpc}$ M-giants sample (filled squares), shows only small variations and in general displays an almost constant scale-height. Interestingly, the last datapoints clustering around $R_{\mathrm{GC}} \simeq 15 \mathrm{kpc}$ show a saturation of the scale-height at $\sim 0.75 \mathrm{kpc}$;

- For Galactocentric distances in the range $8 \leq R_{\mathrm{GC}} \leq$ $25 \mathrm{kpc}$, the $R_{\odot}=16.6 \mathrm{kpc}$ M-giants sample (open squares), shows a bimodal behavior with an: (i) almost constant scale-height within $R_{\mathrm{GC}} \leq 15 \mathrm{kpc}$; and (ii) increasing scale-height for $R_{\mathrm{GC}}>15 \mathrm{kpc}$. The inner radii with the $\sim$ constant trend show a mean scale-height of the same order as that derived from the $R_{\odot}=7.3 \mathrm{kpc}$ M-giants sample. By contrast, at large Galactocentric distances with an increasing scale-height we note that the data-points do not converge to a single value, but show a gradual increase 
Table 1. The limits within which we fit the vertical scale-height for the three M-giants samples. All values are in kpc.

\begin{tabular}{rcc}
\hline \hline Distance & Lower $Z$ limit & Upper $Z$ limit \\
\hline $\mathbf{2 . 8}$ & 0.15 & 0.85 \\
$\mathbf{7 . 3}$ & 0.45 & 2.25 \\
$\mathbf{1 6 . 6}$ & 0.60 & 3.00 \\
\hline
\end{tabular}

with distance out to at least $R_{\mathrm{GC}} \sim 23 \mathrm{kpc}$. The general trends reflect that seen in Alard (2000, starred symbols) and show fair agreement with the Yusifov model. A quantitative parametrization of the observed flare and warp will be subject of a future paper (Momany et al. in prep.).

- In contrast to the almost constant scale-height derived from the $R_{\odot}=7.3$ and $16.6 \mathrm{kpc}$ M-giants samples for the inner regions, the sample at $R_{\odot}=2.8 \mathrm{kpc}$ (open triangles) shows some differences. Between $5 \leq R_{\mathrm{GC}} \leq 9 \mathrm{kpc}$, the $R_{\odot}=2.8 \mathrm{kpc}$ shows a mean scale-height of $0.35 \mathrm{kpc}$, almost half that found for the $R_{\odot}=7.3$ and $16.6 \mathrm{kpc}$ M-giants samples $(0.65 \mathrm{kpc})$.

This difference in scale-height is mainly due to the different weighting of the thin and thick disk populations when extracting the M-giants samples at different distances between $-20^{\circ} \leq b \leq 20^{\circ}$. This translates into different limits when performing the scale-height fitting (cf. Table 1). For the $R_{\odot}=2.8 \mathrm{kpc}$ sample the profile fitting was made between 0.15 and $0.85 \mathrm{kpc}$, clearly more weighted towards the thin disk regime. Reassuringly, the overall mean scaleheight of the $R_{\odot}=2.8 \mathrm{kpc}$ sample $(\sim 0.35)$ is close to typical values for that of the thin disk. On the other hand, for the $R_{\odot}=7.3$ and $16.6 \mathrm{kpc}$ samples the profile fitting starts at $\sim 1.5$ times the thin disk scale-height. Therefore, the overall mean scale-height of the $R_{\odot}=7.3$ and $16.6 \mathrm{kpc}$ samples is an intermediate value $(0.65 \mathrm{kpc})$ that reflects the mixing of the thin and the thick disk populations and respective scale-heights. Lastly, besides the small difference in mean scale-height, one notes that the sample at $R_{\odot}=2.8 \mathrm{kpc}$ shows a similar trend with Galactocentric distance as does the flare model derived by López-Corredoira et al. (2002).

In general, our data-points reflect the fact that the scale-height increases with increasing Galactocentric radii. For the inner Galaxy the scale-height is rather similar to the that in the solar neighborhood, a consistency that continues to about $R_{\mathrm{GC}} \sim$ $15 \mathrm{kpc}$. Further out the scale-height grows relatively rapidly, with a smooth increase until about $\sim 23 \mathrm{kpc}$.

The thickening of the stellar disk is the re-distribution of stars from lower to higher latitudes. A disk thickening or flaring is a phenomenon that requires a reduction below single exponential extrapolation in the stellar density near the Galactic plane for the outer disk. Thus, our detection of (i) a largescale warp signature out to $R_{\mathrm{GC}} \sim 24 \mathrm{kpc}$; and (ii) a disk flaring out to $R_{\mathrm{GC}} \sim 23 \mathrm{kpc}$ provides new evidence of a rather extended Milky Way stellar disk. Therefore, our results add to earlier suggestions by López-Corredoira et al. (2002) and Alard (2000) that the thin disk cut-off at $R_{\mathrm{GC}} \sim 15 \mathrm{kpc}$

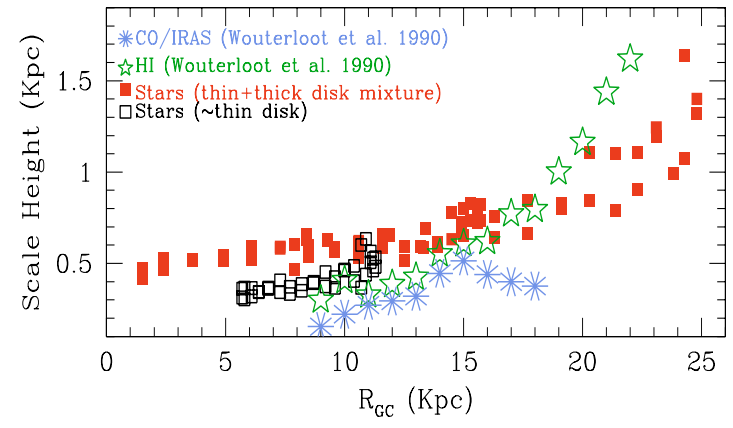

Fig. 15. A comparison of the thickness of the stellar disk, neutral hydrogen gas layer and molecular clouds ensemble for the outer Milky Way. Filled squares show the flaring for the RGB samples at $R_{\odot}=7.3$ and $16.6 \mathrm{kpc}$, whereas the open squares plot the RGB samples at $R_{\odot}=$ $2.8 \mathrm{kpc}$.

(Ruphy et al. 1996) "is not only unnecessary but also inconsistent with the data”.

In Fig. 15 we compare the thickening/flaring of the stellar disk (as obtained from the RGB samples at $R_{\odot}=7.3$ and $16.6 \mathrm{kpc}$ ) with that found for the neutral hydrogen gas layer and molecular clouds ensemble (Wouterloot et al. 1990). On the one hand, the observations by Wouterloot et al. (1990) are a reminder of the presence of molecular clouds with embedded star formation out to $R_{\mathrm{GC}} \sim 20 \mathrm{kpc}$. The authors argue that the lack of CO emission at $R_{\mathrm{GC}}>20 \mathrm{kpc}$ is not to be attributed to the sensitivity of the IRAS survey, rather it shows the absence of recent star formation at these distances. On the other hand, the figure proves the compatibility of the stellar and gaseous flaring for the outer Milky Way. We remind the reader that the filled squares refer to a mixture of thin/thick disk stellar populations, and this explains the gas-stars differences in relative scale-heights. The different populations shown have different vertical velocity dispersions, hence their different scale heights. The only unexpected aspect of this comparison is the very high outer gas scale height, apparently exceeding that of the stars beyond $R_{\mathrm{GC}} \sim 20 \mathrm{kpc}$. This is not understood.

\section{The warped and flared Galactic disk and the connection with stellar over-densities}

Having demonstrated the existence and determined the properties of the stellar warp and flare, we now apply this description of a deformed stellar disk to investigate its impact on reported stellar over-densities at low Galactic latitudes. In the previous sections we showed that the warp and flare are fairly well described by the Yusifov model. We therefore will continue using this model. We remind the reader that the model describes rather well the inner $R_{\mathrm{GC}} \sim 14 \mathrm{kpc}$, but represents only a qualitative description of the outer Milky Way warped and flared regions.

\subsection{The Canis Major over-density}

To show tangibly the importance of allowing for deviations away from symmetry around $b=0^{\circ}$ for studies of the Milky Way stellar disk, in Fig. 16 we show a cut in the YZ plane of 


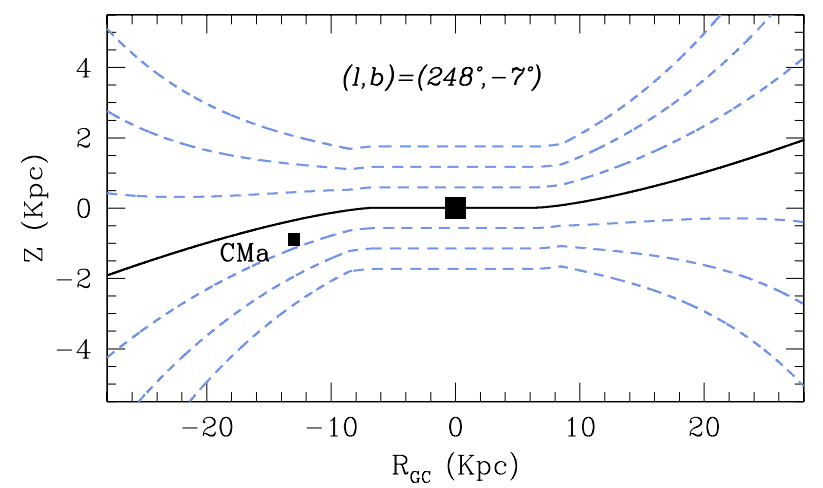

Fig. 16. A cut in the $\mathrm{YZ}$ plane of the warped and flared Galaxy using the Yusifov warp model in the direction and at the distance of CMa. The thick line marks the mean warped stellar disk whereas the grey dashed lines trace the density at $1 \times, 2 \times$ and $3 \times$ the scale-height of the disk.

the warped and flared Galaxy ${ }^{5}$ using the Yusifov warp model in the direction $\left[(l, b)=248^{\circ},-7^{\circ}\right]$ and distance $\left[R_{\odot} \simeq 7.2 \mathrm{kpc}\right]$ of CMa (as most recently derived by Bellazzini et al. 2006).

The solid line traces the mean warped stellar disk, whereas the grey dashed lines follow the three scale heights of the Galactic disk. The figure shows clearly that the CMa overdensity falls at less than $\sim 500 \mathrm{pc}$ from the Galactic warped mid-plane, that is $\sim 50 \%$ the maximum density of the stellar disk at that distance.

This figure clarifies how important is the Galactic warp to any analysis of the CMa over-density. Specifically, comparing star counts at $Z \simeq-1 \mathrm{kpc}$ with those at $Z \simeq+1 \mathrm{kpc}$ one ends up comparing a region falling within $\sim 50 \%$ of the stellar disk density with another having only $\sim 14 \%$.

\subsection{The Monocerous Ring over-densities}

The knowledge of the outer structure of the Galactic disk derived in this analysis does not provide an explanation for the Monocerous Ring. However, we show that the Galactic flare can account for some aspects of some positive detections of the Mon. Ring in various surveys and therefore, needs to be taken into account in investigations of the relative importances of accretion or Galactic structure for the origin for the Mon. Ring.

In Fig. 17 we show the location of positive Mon. Ring detections with respect to the warped and flared Milky Way disk. For the Sloan fields (Newberg et al. 2002), we plot detections at heliocentric distances of 7, 8, 9 and $10 \mathrm{kpc}$, whereas for the INT fields (Conn et al. 2005; and Ibata et al. 2003) we plot the detections at the specific distances given in Table 3 of Conn et al.

Figure 17 shows that all reported Mon. Ring detections are at locations where the structure of the outer warp and flare contribute to the analysis, and must be carefully considered, and at locations where the dynamical interaction between Monocerous and the outer disk will be very important. The Mon. Ring has attributed properties (a radius of $15-20 \mathrm{kpc}$, a

\footnotetext{
${ }^{5}$ See also Fig. 1A and 1B of Evans et al. (1998).
}
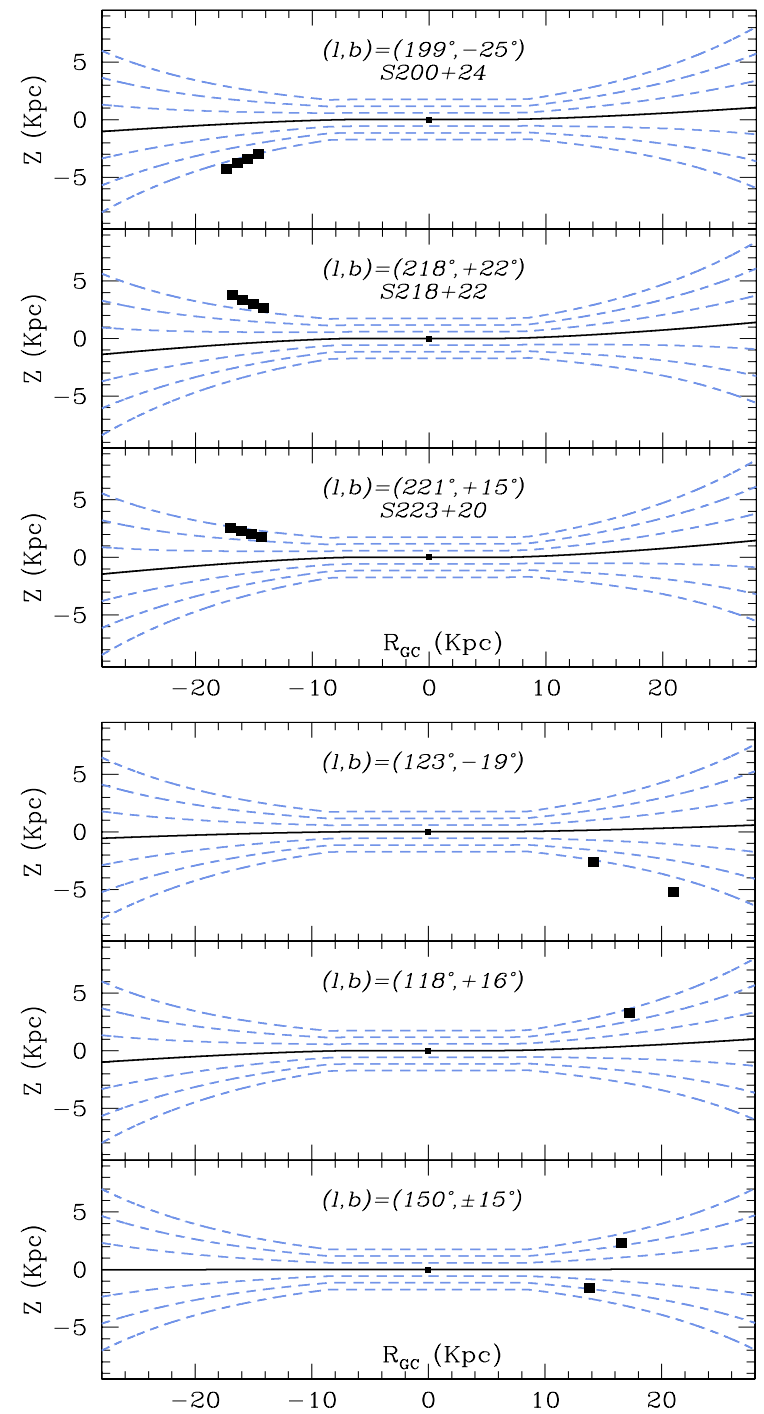

Fig. 17. The same as in Fig. 16 but for fields where positive detections of the Mon. Ring have been reported. For the Sloan detections (upper three panels) we plot detections at heliocentric distances of 7, 8, 9 and $10 \mathrm{kpc}$. Similarly, the lower three panels show INT detections (Conn et al. 2005; Ibata et al. 2003) plotted at the distances given in Table 3 of Conn et al.

radial thickness of $2 \mathrm{kpc}$ and a vertical scale-height of $0.75 \mathrm{kpc}$, Ibata et al. 2003) which place it well within the outer disk defined earlier in this paper.

Of all the Mon. Ring fields, the one at $(l, b)=\left(150^{\circ}, \pm 15^{\circ}\right)$ is perhaps the most important. In their INT survey, Conn et al. (2005) argued that the detection of Mon. Ring structures on both sides of the Galactic plane, at similar Galactic longitudes, is the strongest evidence that the Mon. Ring is not an artefact of Galactic warp effects on star counts. We agree with Conn et al. (2005) that inadequate substraction of the warp is not a valid explanation for Mon. Ring detections above and below the mid-plane. We consider here if disk flaring provides a valid explanation for the Mon. Ring detections in both hemispheres at $(l, b)=\left(150^{\circ}, \pm 15^{\circ}\right)$.

A hint that Mon. Ring detections are related to disk flaring comes from the field at $(l, b)=\left(123^{\circ},-19^{\circ}\right)$, first studied by 
Ibata et al. (2003), who reported a positive Mon. Ring detection at $R_{\mathrm{GC}} \sim 14 \mathrm{kpc}$. This field was re-examined by Conn et al. (2005) who reported a possible new detection at $R_{\mathrm{GC}} \sim 21 \mathrm{kpc}$. Figure 17 shows that the earlier and this new tentative detection of Mon. Ring features follow the expected $Z$ height of the flared MW disk.

We are clearly far from performing a quantitative analysis of the impact of a flared disk on positive detections of Mon. Ring. On the one hand, we should do our best to decrease the rather large uncertainties in the distances of Mon. Ring detections. On the other hand, it would be interesting to have new Galactic models in which, for example, the radial truncation of the stellar disk at $R_{\mathrm{GC}} \sim 14 \mathrm{kpc}$ is dropped, the orientation of the warp phase-angle is updated, and new flare modeling is included. Note for example that the extensive comparison between observed and Besançon simulated diagrams in Mon. Ring fields (Conn et al. 2005) implicitly assume a stellar disk truncation at $R_{\mathrm{GC}} \sim 14 \mathrm{kpc}$. Since most of the Mon. Ring detections are at Galactocentric $>14 \mathrm{kpc}$ it follows that the comparison with the Galactic model is inconsistent.

\section{Galactic structure to $\boldsymbol{R}_{\mathrm{GC}}=\mathbf{2 0} \mathrm{kpc}$ in the outer Galaxy: what do we expect?}

In the previous sections we have shown how complex the outer (beyond the Solar circle) stellar disk of our Galaxy is, and how many intrinsic structures can be identified in it. We have also critically discussed the difficulties in determining the stellar disk main parameters (warp, flare, scale height) by studying its main stellar components. Despite these unavoidable difficulties, we have shown that the parameters inferred from the stellar distribution compare well with what has been obtained from a number of investigations on the distribution of the gas, dust, and neutral Hydrogen. In order to complete our discussion, in this section we add additional information on what we know about the outer Galactic disk, and some information of the properties of the stellar disk in other galaxies.

The outer structure of galactic disks, and the Galactic disk, has been extensively studied for very many years, prior to the current re-discovery of its intrinsic interest. A considerable amount of direct information on the outer Galaxy is available, as is further information by analogy from studies of other galaxies.

A superb review by Burton (1988) summarizes and explains the subject and data, while presenting the observed basic features and results which remain valid in the light of new data. The most extensively studied major component is the $\mathrm{HI}$. This H I extends approximately smoothly and continuously to $R \sim 25$ kpc (Burton 1988 Figs. 7.19, 7.20). The distribution is significantly warped and flared (Figs. 7.18 to 7.23 ), there are significant non-circular motions, and the gas is distributed asymmetrically in azimuth (Fig. 7.21), implying a very significantly lop-sided gas distribution. The results from Burton (1988) were updated and extended by Hartmann \& Burton (1997), and most recently in a very extensive new study of the whole sky H I distribution, using significantly improved calibrations and stray-light corrections, by Kalberla et al. (2005). Inspection of the Hartmann/Burton maps for velocities corresponding to the far outer Galaxy $\left(V_{\mathrm{LSR}} \geq+50 \mathrm{~km} \mathrm{~s}^{-1}\right)$ shows extremely clearly that the outer gas layer exists, is smooth in velocity space (i.e. distance), is centered significantly below the Galactic plane, is lop-sided and warped, and is thick. By contrast, until the present resurgence of interest, there have been rather few large angular-scale studies of the stellar distribution.

A very important point to note in anti-center sight-lines is the considerable "foreground" complexity associated with the spiral arm structure, which extends well beyond the solar circle: in particular, there is the Perseus spiral arm just outside the solar orbit, clearly visible in $\mathrm{CO} l-v$ maps extending across the entire anti-center region from $l \approx 60^{\circ}$ through $180^{\circ}$ to $l \approx 260^{\circ}$. In the anti-center foreground, up to $2 \mathrm{kpc}$ distant, especially in the directions towards $l=140^{\circ}-220^{\circ}$, there are large angular-scale star-forming regions, with their associated dust, including Monoceros, Orion, the Taurus-PerseusAuriga complex, and UMa and Cam OB-star regions. Some of these large-angle structures are physically large in space (e.g. Gould's belt) and are distributed highly asymmetrically with respect to the main Galactic Plane.

Thus the simplest and most robust conclusion from a direct examination of the sky is that one does not expect any simple symmetric distribution of sources on the sky to be an adequate description of the outer Galaxy. One must select tracers by distance, and appreciate complex foregrounds, and expect the outward continuation of normal spiral structure.

\section{1. $\mathrm{HI}$ and $\mathrm{CO}$ surveys: a star-forming disk to $R_{\mathrm{GC}}=20 \mathrm{kpc}$}

Diplas \& Savage (1991), in an early extensive study of outerGalaxy gas which remains valid and topical, conclude: for $12 \leq R_{\mathrm{GC}} \leq 18 \mathrm{kpc}$ the $z$-distribution of the $\mathrm{HI}$ is complicated, and reveals the existence of a confined component and an extended component. The confined component exists only within a Galactocentric radius $R_{\mathrm{GC}} \sim 18-20 \mathrm{kpc}$, while the extended component can be followed in some directions to $R_{\mathrm{GC}} \sim 25-30 \mathrm{kpc}$. In the directions of maximum warp $\left(90^{\circ} \leq l \leq 110^{\circ}\right)$ the average distance of the gas away from the the Galactic plane is found to reach $\sim 4 \mathrm{kpc}$ at $R_{\mathrm{GC}} \sim 24 \mathrm{kpc}$, and the flaring of the layer as measured by $z_{\text {rms }}$ increases to $\sim 3 \mathrm{kpc}$. In these directions the warping outer layer appears to be reliably traced over a region at least $10 \mathrm{kpc}$ thick in $z$. When viewing from the position of the Sun, this implies that the warped and flaring outer Galaxy extends to Galactic latitudes as large as $25^{\circ}$.

This raises the interesting question: is the "confined component" what has been re-discovered recently, the Mon. Ring?

Nakanishi \& Sofue (2004) completed a global analysis of Galactic H I: they show extensions of H I in the outer Galaxy well beyond $20 \mathrm{kpc}$, with evidence of very large scale rotational asymmetry (lop-sided), so much so that the Galaxy extends farther in the first and fourth quadrants. An important point relevant to current discussions is that the general outer distribution of the H I is connected but is not simply smooth. Since the distances are derived from a kinematic model (flat rotation curve), there is a direct degeneracy between kinematic asymmetries - 
i.e. elliptical orbits, and spatial inhomogeneities. Outer spiral structure is evident, as is vertical warping.

We emphasize that from all these and the many other $\mathrm{HI}$ and $\mathrm{CO}$ studies there is no evidence for a significant perturbation from a major satellite accretion event currently underway: quite the opposite in fact, the global approximate smoothness of the $\mathrm{H}$ I distribution is very difficult to reconcile with such a model.

There are many molecular studies of the outer Galaxy indicating that the gas undergoes "normal" outer galaxy rates and distributions of star formation, again supportive of a largely steady-state system. An example is the Brand \& Wouterloot (1995) study of outer IRAS sources, which are star-forming molecular clouds out to $R_{\mathrm{GC}} \simeq 20 \mathrm{kpc}$. They collect data from several earlier studies. Snell et al. (2002) provide a valuable overview of both the Galactic data and studies of other galaxies, concluding that outer Galaxy molecular cloud properties and star formation is a natural extension of the inner Galaxy properties.

We note a study by Freudenreich (1996) of the DIRBE observations of outer galaxy dust, which reports a detection only to $R_{\mathrm{GC}} \simeq 12 \mathrm{kpc}$. It is unclear why this study fails to detect the farther Galactic molecular material, but this paper is the anomaly, all the other studies agree rather well.

A major CO study by Dame et al. (2001) and recent extension to higher latitudes by Dame \& Thaddeus (2004) shows extended $\mathrm{CO}$ emission at latitudes up to $50^{\circ}$ in the anti-center region, with this gas probably being associated with star formation in Taurus-Auriga. Their Fig. 3 shows very well the outer molecular gas at velocities up to $+100 \mathrm{~km} \mathrm{~s}^{-1}$, corresponding to very large Galactocentric distances.

The most recent high-sensitivity southern H I survey, Dickey et al. (2004) shows that the H I warp continues to morenegative $z$-distance as distance increases to a minimum between $R_{\mathrm{GC}}=13-16 \mathrm{kpc}$, with the location a function of longitude through this distance range, and then returns to near $Z=0$ by $R_{\mathrm{GC}}=20 \mathrm{kpc}$. Beyond $20 \mathrm{kpc}$ and out to $30 \mathrm{kpc}$ the H I distribution "is dominated" by a density enhancement which extends coherently over very wide angles, and may be a coherent extension of the Perseus spiral arm for longitudes $l \approx 220^{\circ}-325^{\circ}$. This very large structure, if indeed it is coherent, may be similar to the spiral-like structures in $\mathrm{H}$ I extending well beyond the visible optical disk seen in several other galaxies, with associated star formation and apparently normal structures, by Ferguson et al. (1998) and the several other examples recently observed by UV analysis.

As an example of studies of external spirals, we note the complex, asymmetrical, inflowing H I seen in NGC 2403. Fraternelli et al. (2002) show this is a normal, non-interacting spiral galaxy, suggesting that such $\mathrm{H}$ I features may be common among spiral galaxies and that, perhaps, they have not been detected yet because of the low sensitivity of previous surveys.

\subsection{Expected kinematics}

Bailin \& Steinmetz (2003) note that warps are very common, so much so that perhaps $50 \%$ of galaxies have them
(Reshetnikov \& Combes (1998). But, warps are not long-lived (e.g. Binney 1992), so they must be generated in some way.

Hence, if a warp is kinematically driven, there is no reason to expect simple geometry or kinematics. The actual kinematic pattern will depend on what is the cause, which is not clear. Possible causes include the cumulative effects of many satellites (not necessarily on decaying orbits), though in the case of the Galaxy it may just be the LMC which induces a large-scale reaction from the DM halo (Weinberg 1998; Tsuchiya 2002; but see García-Ruiz et al. 2002) or maybe by Sagittarius (Bailin \& Steinmetz 2003). Bailin \& Steinmetz emphasize that a warp generated by interaction with a satellite is a dynamical warp, so that one does not expect to see stationary or uniformly precessing kinematics. A transient induced warp moves outward at the group velocity of bending waves in a stellar disk, which scales as $\pi G \times \Sigma / \Omega$, for surface density $\Sigma$ and rotational velocity $\Omega$ (Hofner \& Sparke 1994) or $30 \mathrm{kpc} / \mathrm{Gyr}$ near the Sun.

Newberg \& Yanny (2005) suggest significant triaxiality in the large scale structure of the outer galaxy star counts. This is for distances of 3-16 kpc exterior to the Sun. This follows earlier suggestions by Roberta Humphreys. Note that they see similar triaxiality and coherent radial motions in the local old disk, a pattern perhaps consistent with the combined perturbations on circular orbits due to spiral structure (cf. de Simone et al. 2004) and effects due to a bar-shaped perturbation in the disk/bulge potential (cf. Babusiaux \& Gilmore 2005). In any event, simple circular orbits are neither natural nor expected.

\subsection{Expectations from other galaxies}

Star formation in very outer disks, beyond two $R_{25}$, has been detected from $\mathrm{H}$-alpha imaging in several galaxies (e.g. NGC 628, NGC 1058, NGC 6946 Ferguson et al. 1998). Thilker et al. (2005) GALEX imaging the outer disk of M 83, shows current star formation at up to three $R_{\text {Holmberg }}$ in the warped outer disk of M 83. The extreme patchiness of the very outer disk is evident in Fig. 1 of Thilker et al., yet there is no suggestion that what is observed is anything other than star formation in a disk, albeit at very large Galactocentric distances. The GALEX images show star formation, and the existence of both young and intermediate-age open clusters, well beyond an apparent disk "edge". The authors note the location of the young star-forming regions "preferentially on local maxima or filaments in the structure of the warped H I disk", with these complexes superimposed on an extended field stellar population.

Gentile et al. (2003) looked at ESO123-G23, an apparently thin edge-on galaxy with a warp in the optical, and saw H I warped gas up to $15 \mathrm{kpc}$ from the optical major axis, implying a warp through $30^{\circ}$.

An interesting specific study, which reminds us that the dynamics of outer galaxies may not always require current active mergers, is that by Fraternali et al. (2002) of NGC 2403. They studied the spiral galaxy NGC 2403 with the VLA, and discussed the properties of the extended, differentially rotating H I layer with its H I holes, spiral structure, and outer warp. In addition, their new data reveal the presence of a faint, extended, 
and kinematically anomalous component. This shows up in the $\mathrm{HI}$ line profiles as extended wings of emission toward the systemic velocity. In the central regions these wings are very broad (up to $150 \mathrm{~km} \mathrm{~s}^{-1}$ ) and indicate large deviations from circular motion. They separated the anomalous gas component from the cold disk and obtained a separate velocity field and a separate rotation curve for each. The mass of the anomalous component is $1 / 10$ of the total H I mass. The rotation velocity of the anomalous gas is $25-50 \mathrm{~km} \mathrm{~s}^{-1}$ lower than that of the disk. Its velocity field has non-orthogonal major and minor axes that they interpret as due to an overall inflow motion of $10-20 \mathrm{~km} \mathrm{~s}^{-1}$ toward the center of the galaxy. The picture emerging from these observations is that of a cold H I disk surrounded by a thick and clumpy $\mathrm{H}$ I layer characterized by slower rotation and inflow motion toward the center. The origin of this anomalous gas layer is unclear. It is likely, however, that it is related to the high rate of star formation in the disk of NGC 2403 and that its kinematics is the result of a galactic fountain-type of mechanism.

In a related study, Fraternali et al. (2004) show that thin "cold" H I disks are often surrounded by thick layers (halos) of neutral gas with anomalous kinematics. They present results for three galaxies viewed at different inclination angles: NGC 891 (edge-on), NGC 2403 ( $i \simeq 60$ ), and NGC 6946 (almost face-on). These studies show the presence of halo gas up to distances of $10-15 \mathrm{kpc}$ from the plane. Such gas has a mean rotation $25-50 \mathrm{~km} \mathrm{~s}^{-1}$ lower than that of the gas in the plane, and some complexes are detected at very high velocities, up to $200-300 \mathrm{~km} \mathrm{~s}^{-1}$. The nature and origin of this halo gas are poorly understood. It can either be the result of a galactic fountain or of accretion from the intergalactic medium. It is probably analogous to some of the High Velocity Clouds (HVCs) of the Milky Way.

The overall picture from these many studies is that the outer parts of galaxies are complex, asymmetric and time-dependent. While not understood, ongoing mergers of significant companions seem not to be the primary explanation or cause at work.

\section{Discussion and conclusions}

We have used the 2MASS all-sky catalog to probe two important outer Milky Way parameters; the Galactic warp and flare. We show that the Galactic warp can fully explain the detection of the Canis Major over-density. We also show that the Galactic flare of the outer disk will be apparent at those places where there are detection of Monocerous Ring over-densities. Thus, the results presented in this paper provide a reminder that disentangling the accretion history of the outer disk from its complex and time-dependent dynamics remains a challenge. Our results add to the well-known evidence of the complexity of outer Milky Way structure.

The Galactic warp: using 2MASS red clump and red giant stars we first demonstrated the dependency of the warp parametrization (amplitude and phase-angle) on the contamination status of the adopted stellar tracer. Red giants are proved excellent tools in probing the outer most parts of the Milky Way. The detection of a global warp signature by RGB stars at mean heliocentric distances of $R_{\odot}=2.8,7.3$ and $16.6 \mathrm{kpc}$, has helped us understand the following:

- The warp phase-angle is oriented in such a way that at fixed distances from the Sun we observe an asymmetric warp (relatively stronger maximum amplitude in one hemisphere with respect to the other hemisphere). For the $R_{\odot}=2.8$, $7.3 \mathrm{kpc}$ RGB samples, the Southern hemisphere warp maximum shows twice the amplitude of that in the Northern hemisphere. A symmetry of the warp maximum is obtained only for the $R_{\odot}=16.6 \mathrm{kpc}$, at which the dependency on the phase-angle is weakened;

- The warp phase-angle $\left(\phi \simeq+15^{\circ}\right)$ is close to the orientation of the Galactic bar;

- The detection of the stellar warp in the RGB sample at a mean distance $R_{\odot}=2.8 \mathrm{kpc}$ proves that the warp starts within the solar circle;

- The detection of the stellar warp in the RGB sample at a mean distance $R_{\odot}=16.6 \mathrm{kpc}$ proves the presence of an extended Milky Way stellar population out to $R_{\mathrm{GC}} \sim 24 \mathrm{kpc}$. This is in clear contradiction with the assumption of a radial truncation of the stellar disk at $R_{\mathrm{GC}} \sim 14 \mathrm{kpc}$. The detection of an extended stellar disk is confirmed by parallel, and independent studies reporting: (i) recent star formation, traced by molecular clouds, out to $R_{\mathrm{GC}} \sim 20 \mathrm{kpc}$; and (ii) an extended H I spiral arm in the Third and Fourth quadrants;

- For the 3 RGB samples, the Southern hemisphere warp maximum is found around $l \simeq 240^{\circ}$, we therefore establish the identity of the CMa center with the warp maximum.

- The derived stellar warp shows excellent agreement (in both the phase-angle and amplitude of the maximum) with that of the gaseous and interstellar dust distribution. This resolves conflicting evidence of amplitude differences between the 3 Galactic components. The origin of the different warp signatures is due to foreground contamination and distance resolution of the adopted tracer;

These results do not answer the fundamental question of what is the driving mechanism of the warp? There is no general agreement on which process, or processes, generate warps (see Castro-Rodríguez et al. 2002). Satellite accretion remains one among many scenarios put forward to explain warps. Other possibilities include: cosmic infall, bending instabilities, intergalactic magnetic field, accretion of intergalactic medium (see also Olano 2004, for a possible warp-HVCs-Magellanic Clouds connection). Any proposed scenario in a special case should also account for the fact that warps are a ubiquitous property of most, perhaps all, disk galaxies (Sánchez-Saavedra et al. 2003).

The Canis Major Overdensity: we have shown here that $\mathrm{CMa}$ is the southern hemisphere maximum stellar warp. We also note that all CMa properties (measured so far) point to the over-density reflecting a normal warped disk population rather than that of a cannibalized dwarf galaxy. Among these we note:

- the absence of any peculiar radial velocity signature (see Appendix A);

- a possible shortage of an old ( $\geq 9$ Gyr) and metal poor stellar population (see Appendix B); 
- the absence of any peculiar proper motion signature (see Appendix C) and a rather problematic orbital motion 6 ; and - a rather high line-of-sight extent (see Appendix D).

The Galactic flare: having characterized the warp, we also derived the Galactic flare for the 3 RGB samples. Unable to kinematically discern thick from thin disk populations, the retrieved variation with $R_{\mathrm{GC}}$ of the disk thickness shows a rather constant scale-height ( $\sim 0.65 \mathrm{kpc})$ within $R_{\mathrm{GC}} \sim 15 \mathrm{kpc}$. Further out, the mean scale-height increases gradually reaching $\sim 1.5 \mathrm{kpc}$ at $R_{\mathrm{GC}} \sim 23 \mathrm{kpc}$. Whereas this trend refers to a mixture of thin-thick disk populations, for the RGB sample at $R_{\odot} \sim 2.8 \mathrm{kpc}$ (dominated by thin disk populations) we derive a mean scale-height of $\sim 0.35 \mathrm{kpc}$ that is consistent with typical values for the thin disk.

Overall, we trace the stellar disk flaring out to $R_{\mathrm{GC}} \sim$ $23 \mathrm{kpc}$. Thus, we add further and final evidence on the inconsistency of the disk radial truncation hypothesis, at $R_{\mathrm{GC}} \sim 14 \mathrm{kpc}$. Indeed, the detection of stellar disk warping and flaring out to $R_{\mathrm{GC}} \sim 23 \mathrm{kpc}$ is the key answer to the observed drop in stellar density near the Galactic plane.

Acknowledgements. We are indebted to the anymous referee the useful suggestions that improved the overall quality of the paper. YM, GP, LRB and FdA acknowledge the support by the Italian MIUR under the program PRIN03. YM thanks Marcel Clemens and Melissa Evans for a careful reading of the manuscript, and H.I.N.A.S for all the support. This publication makes use of data products from the Two Micron All Sky Survey, which is a joint project of the University of Massachusetts and the Infrared Processing and Analysis Center/California Institute of Technology, funded by the National Aeronautics and Space Administration and the National Science Foundation. We also make use of the WEBDA database and NASA ExtraGalactic Database.

\section{Appendix A: The absence of peculiar CMa radial velocity signature}

In "Why the Canis Major over-density is not due to the Warp", Martin et al. (2004b) presented radial velocities of M-giants at the center of CMa. The overall distribution of radial velocities was strongly characterized by a double-peak morphology. Martin et al. used this distribution to argue that the peak at $V_{\text {rad }}=109 \pm 4 \mathrm{~km} \mathrm{~s}^{-1}$ is to be attributed to an extra population, $\mathrm{CMa}$, that is distinguishable from typical disk stars peaking at $V_{\text {rad }}=61 \pm 4 \mathrm{~km} \mathrm{~s}^{-1}$.

A recent analysis by the same group (Martin et al. 2005) of more than 1500 RGB and RC stars, showed that the bimodal radial velocity distribution was artificially produced by template issues. The new $V_{\text {rad }}$ distribution is now broad; there is no peculiar signature of CMa stars and these are indistinguishable from surrounding disk stars. Nevertheless, Martin et al. proceeded and have identified a "peculiar distance-radial velocity relation that is unlike that expected from thin or thick disk

6 The near circular orbit of CMa is hard to account for in accretion models, usually preferring elliptical orbits. However, Crane et al. (2003) pointed out that this particular issue cannot rule out the accretion scenario since the tidal disruption of satellites depends on whether these satellites were originally born with near-circular orbits (Taffoni et al. 2003). stars". That is, while CMa stars show a mean radial velocity of $72 \pm 7 \mathrm{~km} \mathrm{~s}^{-1}$ at $R_{\odot}=5.5 \mathrm{kpc}$, the mean $V_{\text {rad }}$ increases to about $114 \pm 2 \mathrm{~km} \mathrm{~s}^{-1}$ at $R_{\odot}=8.5 \mathrm{kpc}$.

To date, this "peculiar" $R_{\odot}-V_{\text {rad }}$ relation is considered the strongest kinematic evidence standing in favor of an accretion scenario. However, as we shall demonstrate below, this relation reflects nothing more than the Galactic differential rotation.

To shed more light on this "peculiar" $R_{\odot}-V_{\text {rad }}$ relation, we combine the results obtained by Martin et al. (their Table 2) with a kinematic simulation from the Besançon Galactic model. In the left panel of Fig. A.1 we show the $R_{\odot}-V_{\text {rad }}$ distribution for all simulated stars in the simulated field centered at $(l, b)=\left(240^{\circ},-8^{\circ}\right)$. We remind the reader that the Besançon model imposes a stellar disk truncation already at $R_{\odot}=7.7 \mathrm{kpc}\left(R_{\mathrm{GC}} \simeq 14.0 \mathrm{kpc}\right)^{7}$, more or less at the $\mathrm{CMa}$ distance. Thus, at about $R_{\odot}=7.7 \mathrm{kpc}$ and beyond, one cannot quantitatively compare the observed kinematics with the incomplete Besançon simulation. To highlight the abrupt truncation of the simulated Galactic disk, Thin Disk stars within $R_{\odot}=7.7 \mathrm{kpc}$ are plotted as grey dots, while those further out (Thick Disk and Halo) are plotted as black dots. Before going into more detail, and prior to any selection or analysis, one already sees that there is an intuitable $R_{\odot}-V_{\text {rad }}$ trend in the simulated data, similar to that found in the Martin et al. analysis for CMa stars.

The RC sample of Martin et al. (their Fig. 6) is presented in the left panel (open squares). In order to compare this observed$R C$ sample with an appropriate counterpart from the Besançon simulation (a simulated-RC sample), we extract RC stars from the one square degree, dereddened $K_{0},(J-K)_{0}$ diagram. The simulated color-magnitude diagram is displayed in the upper right panel. In grey symbols we highlight all simulated stellar populations between $5 \leq R_{\odot} \leq 8 \mathrm{kpc}$. The selection box, where we extract simulated-RC stars between $5 \leq R_{\odot} \leq$ $8 \mathrm{kpc}$ is shown as grey rectangle area. We emphasize that this simulated-RC sample, as that studied by Martin et al., is subject to foreground contamination.

The advantage of using simulated data is that one can easily isolate the foreground dwarf contamination from the simulated$R C$ sample, by means of the simulated stars distance entries. All stars included in the simulated-RC sample are plotted in the left panel (open triangles), showing clearly the contamination by local dwarfs within $\sim 1 \mathrm{kpc}$. Between 5.5 and $8.0 \mathrm{kpc}$ simulated-RC stars overlap with the Martin et al. observed sample (a colored version of the figure is more appropriate for the disentangling).

The middle right panel shows the radial velocities distribution of the Martin et al. observed-RC sample (dashed histogram). The distribution of simulated-RC stars as extracted from the Besançon simulation is plotted as a continuous histogram. We make use of the Martin et al. (2005) estimate of the $V_{\text {rad }}$ intrinsic dispersion of disk stars at the CMa distance and add this value (via a Gaussian distribution) to the simulated-RC stars. The model histogram has been scaled to

\footnotetext{
7 As demonstrated in Sect. 5, the results presented in this paper confirm earlier suggestions that a disk truncation hypothesis at $R_{\mathrm{GC}} \simeq 14.0$ is neither needed nor justified.
} 


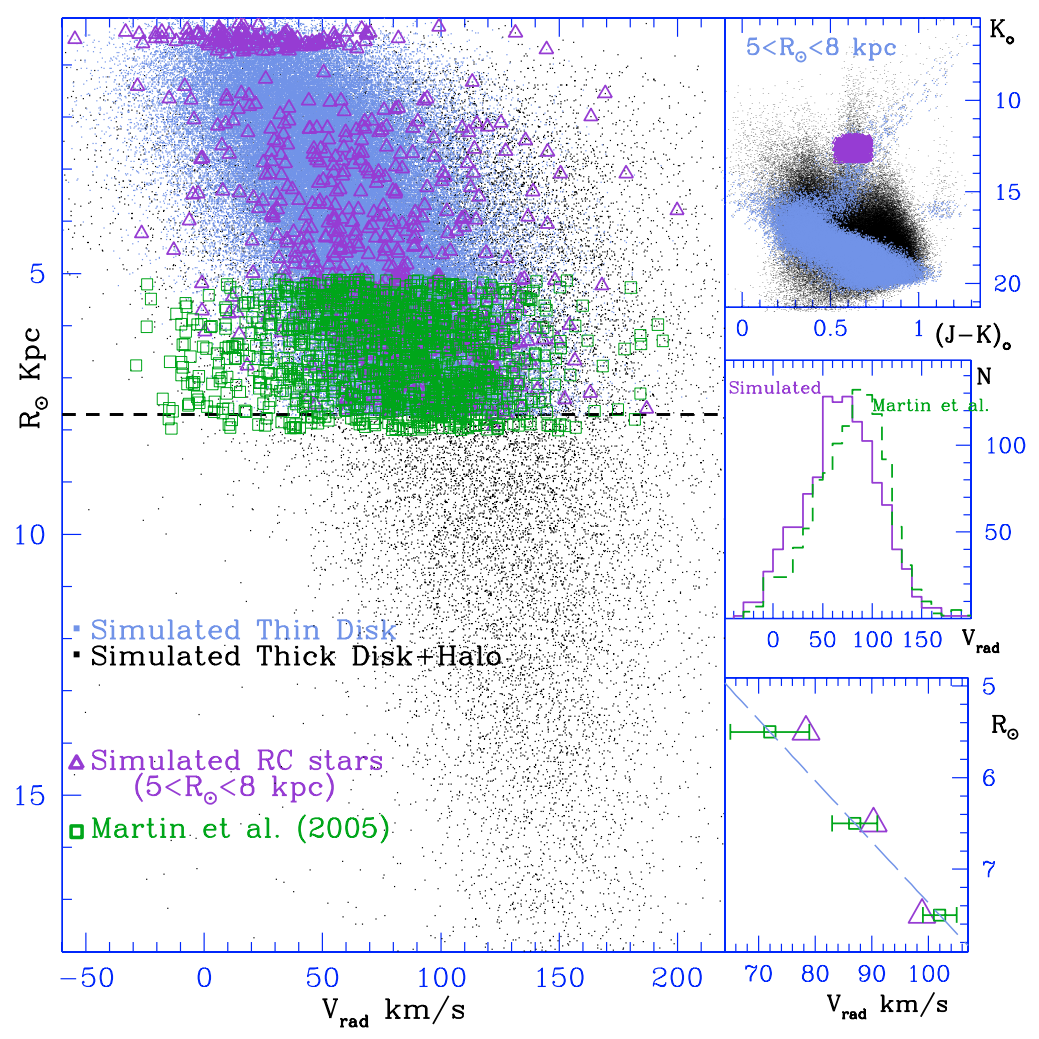

Fig. A.1. Explaining the "peculiar" $R_{\odot}-V_{\text {rad }}$ relation found for CMa stars as due to Galactic differential rotation. The left panel shows a Besançon simulated $R_{\odot}-V_{\text {rad }}$ distribution for stars at the CMa center. Grey dots highlight the modeled Thin Disk truncation at $R_{\odot}=7.7 \mathrm{kpc}$ (marked by a horizontal line). Black dots plot the Thick Disk and Halo stars. Open triangles show the $R_{\odot}-V_{\text {rad }}$ distribuition for simulated stars extracted from the synthetic color-magnitude diagram (upper right panel), and falling within a box delimiting the RC region between $5 \leq R_{\odot} \leq 8 \mathrm{kpc}$. The open squares are the observed-RC sample from Martin et al. (their Fig. 6). Right middle panel shows the distribution of radial velocities of the Martin et al. observed-RC sample (dashed histogram), and the scaled distribution of simulated-RC stars (continuous histogram). The right lower panel shows the mean $V_{\text {rad }}$ of the $d w a r f s$-decontaminated and $R C$-simulated sample (open triangles) estimated in three distance intervals. The open squares plot the observed mean $V_{\text {rad }}$ values for CMa RC stars (see Table 2 of Martin et al.) in the same distance intervals. The error bars are from the same table. The Dashed line is a linear fit to the Martin et al. mean values; which is fully compatible with recent estimates of the $A$ Oort constant being $A=17.23 \pm 0.35$.

fit the observed histogram: other appropriate scaling is not possible since the Martin et al. (2005) selection function of RC stars is unknown. The similarity of the two distributions is remarkable, we note however that the histogram of simulated$R C$ stars shows a slightly higher distribution (with respect to the observed sample) for stars with $V_{\mathrm{rad}}<80 \mathrm{~km} \mathrm{~s}^{-1}$, and a lower distribution for $V_{\text {rad }}>80 \mathrm{~km} \mathrm{~s}^{-1}$. The later feature can be explained as being due to the truncation of disk population in the Besançon model at $R_{\odot}=7.7 \mathrm{kpc}$ : simulating more distant populations will result in more stars only having $V_{\text {rad }}$ higher than $80 \mathrm{~km} \mathrm{~s}^{-1}$. On the other hand, the higher distribution of simulated-RC stars at $V_{\mathrm{rad}}<80 \mathrm{~km} \mathrm{~s}^{-1}$ can be due to a stronger foreground dwarf contamination in the input model (note in fact the discrete and distinguishable distribution of local dwarfs within $1 \mathrm{kpc}$ ). The two distributions remain, however, very similar and this is further demonstrated in the lower right panel where we (i) exclude nearby dwarfs from the simulated-RC sample, (ii) use this cleaned simulated$R C$ sample to estimate the mean $V_{\text {rad }}$ in 3 distances intervals $\left(5 \leq R_{\odot} \leq 6,6 \leq R_{\odot} \leq 7\right.$ and $\left.7 \leq R_{\odot} \leq 8 \mathrm{kpc}\right)$; and (iii) compare the cleaned and simulated $V_{\text {rad }}$ values with those derived by Martin et al. (2005), in the 3 distance intervals.
We emphasize the fact that the mean CMa $V_{\text {rad }}$ values derived in the 3 distance intervals (Martin et al 2005, open squares in the lower right panel) are based on what they call "CMa RC sample", which they disentangle from the "contaminating population". Keeping in mind all the uncertainties associated with the Besançon model, one clearly sees that the cleaned simulated-RC sample (open triangles in the lower right panel) shows, within $l \sigma$, the same $R_{\odot}-V_{\text {rad }}$ trend found by Martin et al. for a cleaned CMa RC sample.

One last piece of evidence which demonstrates that the "peculiar CMa observed $R_{\odot}-V_{\text {rad }}$ trend" is nothing but the imprint of Galactic differential rotation of normal disk stars is shown in the lower panel of Fig. A.1. Indeed, a linear fit of the three points reported by Martin et al. (2005) yields $R_{\odot}=0.067 \times V_{\mathrm{rad}}$. Using the Oort equation:

$$
\begin{aligned}
& V_{\mathrm{rad}}=A \times R_{\odot} \times \sin \left(2 \times l^{\circ}\right) \\
& V_{\mathrm{rad}}=A \times \sin \left(2 \times 240^{\circ}\right) \times R_{\odot}=(1 / 0.067) \times R_{\odot}
\end{aligned}
$$

one easily gets $A=17.23 \pm 0.35$, fully compatible with recent estimates of the $A$ Oort constant (see Dehnen 1998). Obviously, one has to consider the CMa large heliocentric distance and 
location with respect to the Galactic disk, however, the linear relation is really surprising, and does not leave room for any other easy interpretation.

Given that (i) the bimodal $V_{\text {rad }}$ distribution (Martin et al. 2004b) was due to an artifact in their reduction procedure, and (ii) that the claimed peculiar $R_{\odot}-V_{\text {rad }}$ relation (Martin et al. 2005) is well reproduced by a self-consistent kinematical model, and most importantly, seems to be nothing more than the imprint of differential Galactic rotation. We therefore conclude that the stellar populations of CMa do not show any peculiar or distinguishable $V_{\mathrm{rad}}$ signature with respect to normal disk stars.

\section{Appendix B: The possible shortage of an old ( $\geq 9 \mathrm{Gyr}$ ) and metal poor CMa stellar population}

All studied dwarf galaxies are known to possess an ancient stellar population that is traced by the detection of either $\geq 9 \mathrm{Gyr}$ RR Lyrae variables and/or red/blue horizontal branch stars of similar age. Dwarf galaxies in the Local Group show no exception to this rule (Mateo 1998; Tolstoy 2000; Grebel 2001) ${ }^{8}$. To this adds the most recent entry, Ursa Major (Willman et al. 2005), which shows a clear clump of blue horizontal branch stars. Those in favor of an extra-Galactic accretion scenario of CMa often highlight the similarities (e.g. mean metallicity, mean mass, $M_{V}$ etc.) between the CMa stellar populations and the Sagittarius dwarf spheroidal. As a consequence, it is particularly important to investigate the presence of an old ( $\geq 9 \mathrm{Gyr}$ ) and metal-poor population of CMa.

Needless to say, an old and metal-poor population is necessary to metal enrich successive generations of stars, otherwise one cannot explain how a metal-rich galaxy $([\mathrm{Fe} / \mathrm{H}] \geq-1)$ could have built metal-poor clusters. The Sagittarius dwarf fulfills this requirement; i.e. besides being on average a metal-rich galaxy, it possesses a metal-poor component that is traced by RR Lyraes and blue horizontal branch stars (see Monaco et al. 2003 and references therein), and possibly extreme horizontal branch stars (Momany et al. 2004a). This explains the progeny of metal-poor clusters like Terzan 8 and M54. Studying the Sagittarius stellar populations outside the tidal radius of M 54, Monaco et al. (2003) proved that the group of blue horizontal branch stars identified in S341+57-22.5 by Newberg et al. (2002), are fully compatible with the blue horizontal branch of Sagittarius. This shows that a stream, narrow by definition, originating from a disrupting dwarf is likely to leave a footprint of all its stellar populations (both red and blue).

Despite the fact that contamination can seriously hamper the identification of old blue horizontal branch stars near the Galactic plane, we note that the CMa over-density has not been associated with a clear identification, or even a hint, of old blue horizontal branch stars. Figure B.1 displays a widefield color-magnitude diagram of the CMa center, based on

${ }^{8}$ Izotov \& Thuan (2004) proposed a scenario in which young ( 500 Myr) galaxies like IZw 18 are still being born in today's universe. Nevertheless, in Momany et al. (2005) we showed that $\mathrm{IZw} 18$ can be much older and that, at the moment, there is no photometric evidence of the existence of young galaxies.

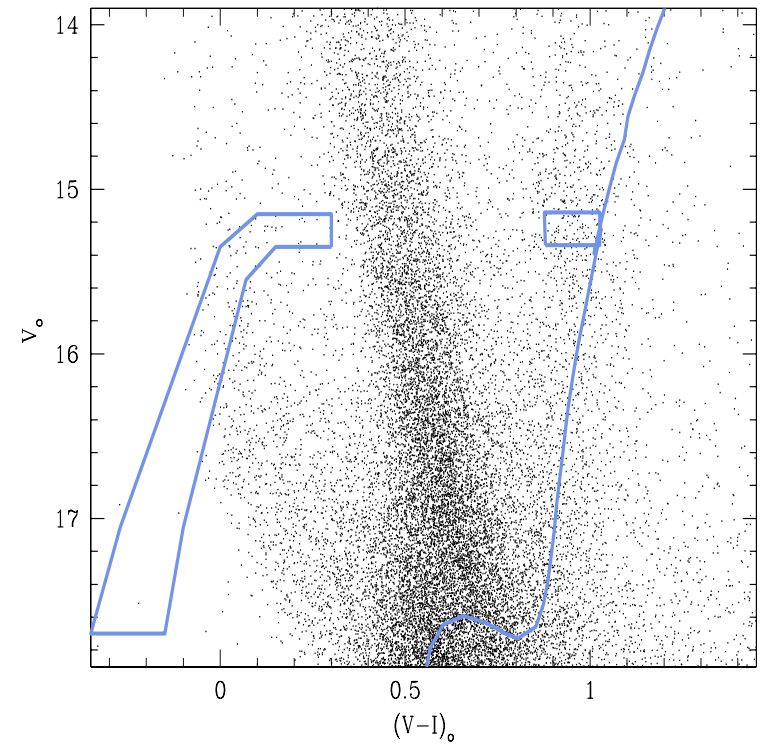

Fig. B.1. A $1^{\circ}$ dereddened color-magnitude diagrams of the core of CMa. Over-plotted is a $6.3 \mathrm{Gyr} Z=0.004([\mathrm{Fe} / \mathrm{H}] \simeq-0.76)$ isochrone from The Girardi et al. (2002) library. The assumed distance modulus is $(m-M)_{0}=14.5$. Each star was dereddened according to its position in the Schlegel et al. (1998) reddening maps. Also plotted are the red clump and blue horizontal branch location of the Sagittarius, as derived by Monaco et al. (2003).

archival ESO/2.2 m telescope data. The data has been reduced and calibrated following the standard techniques presented in (Momany et al. 2001). Upon this color-magnitude diagram we over-plot the mean location of red clump and blue horizontal branch stars in the Sagittarius dwarf (as derived in Monaco et al. 2003), shifted to match the CMa red clump population. No hint of an over-density of stars at the expected blue horizontal branch location can be seen. Instead, the distribution of stars between $14.0 \leq V_{0} \leq 16.5$ and $(V-I)_{0} \leq 0.3$ smoothly increases towards fainter magnitudes. We remind the reader that at $V_{0} \simeq 16.0$, and in between $0.0 \leq(V-I)_{0} \leq 0.5$ there lies a population of young $\leq 100 \mathrm{Myr}$ main sequences stars which belong to the Norma-Cygnus spiral arm, as demonstrated by Carraro et al. (2005) by means of $U B V$ two-color diagrams. This population is intermediate between the disk MS stars (oblique sequence extending from $(V-I)_{0} \simeq 0.2$ at $V_{0} \simeq 12.0$ to $(V-I)_{0} \simeq 0.6$ at $\left.V_{0} \simeq 18.0\right)$ and the blue plume population at bluer colors. An eventual over-density due to the presence of blue horizontal branch star $\left(7000 \leq T_{\text {eff }} \leq 11000 \mathrm{~K}\right)$ should take place in between the disk main sequence and blue plume, but this region lacks any significant over-density. A similar indication is found in the optical color-magnitude diagrams of Martínez-Delgado (2005) and Bellazzini (2006).

Recently, Mateu et al. (2004) reported on a preliminary RR Lyrae search in an area of 8.3 square degrees at the center of the CMa. Five RR Lyraes were found at heliocentric distances of less than $6.5 \mathrm{kpc}$. Assuming the most recent CMa distance range $(6.2-8.2 \mathrm{kpc}$ by Bellazzini et al. 2006) the 5 variables with a mean distance of $5.6 \mathrm{kpc}$ are outside the main body of the CMa over-density. Yet, as noted by the Mateu et al., the detection of 5 variables is higher than what would be 
expected in the same volume of the Galactic Halo ( $\sim 1$ variable). Unfortunately, a direct comparison with the expected number of Disk RR Lyrae is difficult since the distribution (and density) of RR Lyrae stars in the thick disk is not known. Moreover, one should add the current uncertainty in (i) disentangling Disk and Halo RR Lyrae at low Galactic latitudes, and (ii) the disk density profile as a function of $R_{\mathrm{GC}}$. As demonstrated thoroughly in this paper, the disk radial extension does not show an abrupt truncation at $R_{\mathrm{GC}} \sim 14 \mathrm{kpc}$, and this might increase the number of expected variable stars.

We therefore conclude that there is no evidence which demonstrates the presence of an old, blue horizontal branch population CMa. At the same time, RR Lyrae surveys leave this issue rather 2open, highlighting intrinsic difficulties (expected Galactic contamination and relative vicinity of CMa) of similar studies. More data is needed to draw a firmer conclusion.

\section{Appendix C: The negative vertical velocity of the Canis Major}

Dinescu et al. (2005) have recently re-measured the absolute proper motion at the center of the Canis Major over-density, which was first determined by Momany et al. (2004b using UCAC2 catalogs ${ }^{9}$ ). The authors find that while CMa has an inplane rotation (similar to the mean of thick disk stars) it shows significant $3 \sigma$ motion perpendicular to the disk. In turn, they find this inconsistent (at a $7 \sigma$ level) with the expected motion of the warp at these Galactic locations (estimated in Drimmel et al. 2000). This incompatibility lead Dinescu et al. (2005) to conclude that CMa is part of a satellite galaxy remnant.

To resolve the claimed incompatibility we note that the Dinescu et al. (2005) conclusions rely on two points. The first is the assumption that their selected sample [used to measure the proper motion and consisting of "likely main sequence stars" or blue plume stars] is a photometrically clean sample of genuine CMa stars, un-contaminated by Galactic disk stars. This assumption however is in complete contradiction with the recent finding of Carraro et al. (2005), who have shown that the distribution of the blue plume population in the background of 30 open clusters (in the Third quadrant and at $R_{\odot} \geq 7 \mathrm{kpc}$ ) follows the expected pattern of the Norma-Cygnus (outer) spiral arm remarkably well. Thus the CMa over-density resides on an outer spiral arm, and the young blue plume population (seen in various CMa diagrams) is simply not associated with it. This is a particularly important issue because it explains the difference in the proper motion at the CMa-center obtained by Dinescu et al. (2005, using MS stars) and Momany et al. (2004b, using RGB stars) which in turn brings us back to the situation where the proper motion of CMa is indistinguishable from surrounding disk stars ${ }^{10}$.

\footnotetext{
9 See however Momany \& Zaggia (2005) reporting the presence of possible systematic errors in the UCAC catalog.

${ }^{10}$ Note also that in calculating $W$, Dinescu et al. (i) apply a mean $V_{\text {rad }}$ value referring to the artificially produced $V_{\text {rad }}$ peak at $109 \pm 4 \mathrm{~km} \mathrm{~s}^{-1}$ by Martin et al. (2004b); (ii) assume a distance of $8.1 \mathrm{kpc}$, different from the most recent determination $(7.2 \mathrm{kpc})$ by Bellazzini et al. (2006); and (iii) combine the proper motion based on blue plume stars with $V_{\text {rad }}$ values that are based on red clump stars.
}

Secondly, the fact that the negative $W$ of CMa is irreconcilable with the expected signature of the Galactic warp (positive $W$ ) is simply not true, since these expectations are modeldependent. We will demonstrate this by using the same cited article of Drimmel et al. (2000), paying more attention to what these authors report at the end of their article.

We first remind the reader that Drimmel et al. (2000) used Hipparcos OB stars as tracers of the Galactic warp. Therefore, it is particularly important to recall recent problems in the inferred Hipparcos parallaxes of O stars. Schröder et al. (2004) using a sample of 153 stars, conclude that their absolute magnitude (calculated from their apparent magnitude and the Hipparcos parallax) appear to be much fainter than expected, by up to $5 \mathrm{mag}$. Such huge differences were found to arise from the distances at which $\mathrm{O}$ stars were located, and the level of precision of the parallax measurements achieved by Hipparcos. Their Fig. 2 clearly shows that large magnitude differences are expected when using the relatively uncertain Hipparcos parallaxes at distances of already $\sim 1 \mathrm{kpc}$.

Accounting for this fact, we reconsider the work of Drimmel et al. (2000). In their Fig. 11, they report a comparison of the measured vertical velocity component $(W)$ vs. Galactocentric distances. At $R_{\mathrm{GC}} \simeq 8.5 \mathrm{kpc}$, a divergence between measured $W$ values of distant OB stars (increasingly negative $W$ values) and the expected observable signature of the warp (increasingly positive $W$ values) occurs. It remains, however, that Drimmel et al. (2000, their Sect. 7) investigate the effect of bias, amplitude and precision on the inconsistency between the observed and the expected warp $W$ values. In their Fig. 12, the authors unambiguously show that "negative vertical motions are finally achieved", if they allow for: (i) a warp that has half the amplitude with respect to the one they derive; (2) a warp precessing at $-25 \mathrm{~km} \mathrm{~s}^{-1} \mathrm{kpc}^{-1}$; and (iii) a $0.5 \mathrm{mag}$ error is added.

Now, leaving aside the excessively high precision rates which Drimmel et al. (2000) also find questionable, it is a matter of fact that the remaining two bias strongly contribute in reproducing negative vertical velocities. On the one hand, the results presented in this paper (see in particular Fig. 2 of Yusifov 2004) show that the warp amplitude as derived by Drimmel et al. (2000) is very high. Indeed, at a Galactocentric distance of $10 \mathrm{kpc}$, Drimmel et al. note that their warp amplitude is more than twice the gas warp amplitude, and not surprisingly they halve it. In particular, the authors note that the effect of misplacement of a star to larger distances is that its measured relative vertical motion will be smaller than its true relative vertical motion.

On the other hand, there is now enough evidence to suggest that the Hipparcos data can have significantly higher errors as suggested by Schröder et al. (see also Soderblom et al. (2005) on a recent confirmation of errors in the Hipparcos parallaxes already at the Pleiades distance). Thus, introducing a 0.5 mag (as done by Drimmel et al. 2000) could have been seen as extreme at that time. Nowadays, however, with confirmed errors of more than 2 mag (for the same sample of hot stars) at much closer distances, the evidence suggests that the expected warp signature can be and is compatible with negative vertical velocity. 
Since Dinescu et al. mistake main sequence stars belonging to a Galactic spiral arm for genuine CMa population, and given the above discussion on the compatibility of negative $W$ values with the warp at $\mathrm{CMa}$ location, we conclude that the proper motion of CMa does not show any peculiar signature with respect to Galactic disk stars.

\section{Appendix D: The "narrowness" of CMa main sequence}

Recently Martínez-Delgado et al. (2005) presented a deep wide-field $B,(B-R)$ color-magnitude diagram of the $\mathrm{CMa}$ center and derived the line-of-sight extent (or depth) of CMa. For this task, Martínez-Delgado et al. estimated the observed width of the CMa main sequence $\left(\sigma_{\text {MS total }}^{B}\right)$ by selecting stars in a narrow color range, $1.5 \leq(B-R) \leq 1.55$, at $B \simeq 22.1$. Their best fitting of $\sigma_{\text {MS,total }}^{B}$ (converted into kilo-parsec) yielded $F W H M=1.95 \mathrm{kpc}$. Moreover, and for a proper comparison with other studied Local Group dwarf spheroidals, they also estimated the line-of-sight half-brightness radius $\left(r_{1 / 2}\right)$, obtaining $\sim 1 \mathrm{kpc}$.

Martínez-Delgado et al. (2005) noted that this size is "significantly bigger than that of several dwarf galaxies in the Local Group", e.g. the $r_{1 / 2}$ of the Fornax dwarf spheroidal is $0.33 \mathrm{kpc}$ (see Table 4 of Irwin \& Hatzidimitriou 1995). Nevertheless, one finds that this rather large value found by Martínez-Delgado et al. (2005) is often cited in support of a "narrow extent line-of-sight" of CMa (Martin et al. 2005; Conn et al. 2005) or even evidence of a "typical size of a dwarf spheroidal galaxy" (Bellazzini et al. 2006).

In regards to this, and in light of recent finding of the presence of the Norma-Cygnus (outer) spiral arm in the third quadrant (Carraro et al. 2005), we compare the $F W H M$ of CMa as obtained by Martínez-Delgado et al. (2005) with typical values for Galactic spiral arms. Following Bronfman et al. (2000), we estimate the $F W H M$ of the most distant spiral arm at $R / R_{0}=1.3$, where $R_{0}=8.5 \mathrm{kpc}$. Relying on their Fig. 8, we estimate a $F W H M$ of $\sim 1.7 \mathrm{kpc}$, very much in accordance with the Martínez-Delgado et al. (2005) $1.95 \mathrm{kpc}$ value for CMa. Indeed, one must also bear in mind that an outer spiral arm would also be more flared, thereby a FWHM of $\sim 1.7 \mathrm{kpc}$ sets only a lower limit. One last piece of evidence that the reported CMa $F W H M$ is compatible with a spiral arm feature comes from the study by McClure-Griffiths et al. (2005). The detected H I arm in the Fourth and Third quadrants appeared to be approximately 1 to $2 \mathrm{kpc}$ thick along the line of sight.

We therefore conclude that the CMa depth as measured by Martínez-Delgado et al. (2005) is more compatible with a distant spiral arm rather than a dwarf spheroidal.

\section{Note added in proof}

\section{Appendix E: CMa and the Orion arm connection}

Recently, Moitinho et al. (2006) used young ( 100 Myr) open clusters and field "blue plume" population to reconstruct the spiral structure in the Third Galactic quadrant. They confirmed the detection of a structure interpreted as a natural extension of the Norma-Cygnus spiral arm. Their conclusion (based on UBVRI photometry of OB objects) is consistent with our finding of an extended MW disk (using 2MASS M-giants). Moitinho et al. went a step further and proposed that the CMa M-giant over-density is simply the result of looking along the extension of the local Orion arm in the Third quadrant.

In regards to this, we note that the Moitinho et al. (2006) and Carraro et al. (2005) papers have been valuable in clearing the connection between the blue plume population (erroneously attributed to CMa by Martínez-Delgado et al. 2005; Bellazzini et al. 2004; and Dinescu et al. 2005) and both the red clump and red giant populations (that first brought to the detection of the CMa over-density). Nevertheless, the Moitinho et al. analysis does not address, nor preclude, the very occurrence of the CMa over-density at $b \sim-8^{\circ}$. Indeed, the detection of a spiral arm composed by young blue objects (best identified in $U B V$ photometry) is not sufficient to affirm/conclude a Galactic nature for the un-ambiguous presence of the older (2-10 Gyr) CMa M-giant over-density (best seen in the 2MASS infrared photometry). The asymmetry in the distribution of the MW disk stars (Galactic warp) remains the explanation for the observed asymmetric number density at $b \sim-8^{\circ}$ and $b \sim+8^{\circ}$. The warp signature is evident in Fig. 2 of Moitinho et al. (2006).

\section{References}

Alard, C. 2000 [arXiv: astro-ph/0007013]

Amôres, E. B., \& Lépine, J. R. D. 2005, AJ, 130, 659

Bailin, J., \& Steinmetz, M. 2003, Proceedings of the conference, Satellites and Tidal Streams [arXiv: astro-ph/0310199]

Babusiaux, C., \& Gilmore, G. 2005, MNRAS, 358, 1309

Benjamin, R. A., Churchwell, E., Babler, B. L., et al. 2005, ApJ, 630, L149

Bensby, T., Feltzing, S., \& Lundström, I. 2004, A\&A, 421, 969

Bellazzini, M., Ibata, R., Monaco, L., et al. 2004, MNRAS, 354, 1263 [B04]

Bellazzini, M., Ibata, R., Martin, N., et al. 2006, MNRAS, 155 [arXiv: astro-ph/0504494]

Binney, J. 1992, ARA\&A, 30, 51

Bonifacio, P., Monai, S., \& Beers, T. C. 2000, AJ, 120, 2065

Brand, J., \& Wouterloot, J. G. A. 1995, A\&A, 303, 851

Bronfman, L., Casassus, S., May, J., \& Nyman, L.-Å. 2000, A\&A, 358,521

Burton, W. B. 1988, in Galactic and Extragalactic Radio astronomy, ed. G. L. Verscuur, \& K. I. kellermann (Springer)

Carney, B. W., \& Seitzer, P. 1993, AJ, 105, 212

Carraro, G., Vázquez, R. A., Moitinho, A., \& Baume, G. 2005, ApJ, 630, L153

Carraro, G., Bresolin, F., Villanova, S., et al. 2004, AJ, 128, 1676

Castro-Rodríguez, N., López-Corredoira, M., Sánchez-Saavedra, M. L., \& Battaner, E. 2002, A\&A, 391, 519

Conn, B. C., Lewis, G. F., Irwin, M. J., et al. 2005, MNRAS, 362, 475

Crane, J. D., Majewski, S. R., Rocha-Pinto, H. J., et al. 2003, ApJ, 594, L119

Cutri, R. M., et al. 2003, VizieR Online Data Catalog, 2246

Dame, T. M., Hartmann, D., \& Thaddeus, P. 2001, ApJ, 547, 792

Dame, T. M., \& Thaddeus, P. 2004, Milky Way Surveys: The Structure and Evolution of our Galaxy, ASP Conf. Ser., 317, 66

De Simone, R., Wu, X., \& Tremaine, S. 2004, MNRAS, 350, 627

Dehnen, W. 1998, AJ, 115, 2384

Dickey, J. M., McClure-Griffiths, N. M., Gaensler, B. M., \& Green, A. J. 2004, Milky Way Surveys: The Structure and Evolution of our Galaxy, ASP Conf. Ser., 317, 26

Dinescu, D. I., Martínez-Delgado, D., Girard, T. M., et al. 2005, ApJ, 631, L49

Diplas, A., \& Savage, B. D. 1991, ApJ, 377, 126 
Djorgovski, S., \& Sosin, C. 1989, ApJ, 341, L13

Drimmel, R., Smart, R. L., \& Lattanzi, M. G. 2000, A\&A, 354, 67

Drimmel, R., \& Spergel, D. N. 2001, ApJ, 556, 181

Drimmel, R. 2005, The Three-Dimensional Universe with Gaia, ESA SP-576, 167

Evans, N., Gyuk, G., Turner, M., \& Binney, J. 1998, ApJ, 501, L45

Ferguson, A. M. N., Wyse, R. F. G., Gallagher, J. S., \& Hunter, D. A. 1998, ApJ, 506, L19

Fraternali, F., van Moorsel, G., Sancisi, R., \& Oosterloo, T. 2002, AJ, 123,3124

Fraternali, F., Oosterloo, T., Boomsma, R., Swaters, R., \& Sancisi, R. 2004, IAU Symp., 217, 136

Freudenreich, H. T., et al. 1994, ApJ, 429, L69

Freudenreich, H. T. 1996, ApJ, 468, 663

Freudenreich, H. T. 1998, ApJ, 492, 495

Frinchaboy, P. M., Majewski, S. R., Crane, J. D., et al. 2004, ApJ, 602, L21

García-Ruiz, I., Kuijken, K., \& Dubinski, J. 2002, MNRAS, 337, 459

Gentile, G., Fraternali, K. U., \& Salucci, P. 2003, A\&A, 405, 969

Girardi, L., Bertelli, G., Bressan, A., et al. 2002, A\&A, 391, 195

Grebel, E. 2001, Ap\&SS, 277, 231

Hartmann, Dap, \& Burton, W. B. 1997 in Atlas of galactic neutral hydrogen (Cambridge, New York: Cambridge University Press)

Helmi, A., Navarro, J. F., Meza, A., Steinmetz, M., \& Eke, V. R. 2003, ApJ, 592, L25

Hofner, P., \& Sparke, L. S. 1994, ApJ, 428, 466

Ibata, R. A., Irwin, M. J., Lewis, G. F., Ferguson, A. M. N., \& Tanvir, N. 2003, MNRAS, 340, L21

Irwin, M., \& Hatzidimitriou, D. 1995, MNRAS, 277, 1354

Izotov, Y. I., \& Thuan, T. X. 2004, ApJ, 616, 768

Kalberla, P. M. W., Burton, W. B., Hartmann, D., et al. 2005, A\&A, 440,775

López-Corredoira, M., Cabrera-Lavers, A., Garzón, F., \& Hammersley, P. L. 2002, A\&A, 394, 883

Majewski, S. R., Skrutskie, M. F., Weinberg, M. D., \& Ostheimer, J. C. 2003, ApJ, 599, 1082

Majewski, S. R., Ostheimer, J. C., Rocha-Pinto, H. J., et al. 2004, ApJ, 615,738

Manchester, R. N., Hobbs, G. B., Teoh, A., \& Hobbs, M. 2005, AJ, 129,1993

Martin, N., Ibata, R., Bellazzini, M., et al. 2004a, MNRAS, 348, 12 [M04a]

Martin, N. F., Ibata, R., Conn, B., et al. 2004b, MNRAS, 355, L33 [M04b]

Martin, N. F., Ibata, R. A., Conn, B. C., et al. 2005, MNRAS, 362, 906

Martínez-Delgado, D., Butler, D. J., Rix, H.-W., et al. 2005, ApJ, 633, 205

Mateo, M. L. 1998, ARA\&A, 36, 435

Mateu, C. E., Vivas, A. K., Zinn, R., \& Miller, L. 2004 [arXiv:astro-ph/0504333]

McClure-Griffiths, N. M., Dickey, J. M., Gaensler, B. M., \& Green, A. J. 2004, ApJ, 607, L127

McClure-Griffiths, N. M., Dickey, J. M., Gaensler, B. M., et al. 2005, ApJS, 158, 178

Moitinho, A., Vázquez, R. A., Carraro, G., et al. 2005, MNRAS [arXiv:astro-ph/0602493]
Momany, Y., Vandame, B., Zaggia, S., et al. 2001, A\&A, 379, 436

Momany, Y., Bedin, L. R., Cassisi, S., et al. 2004a, A\&A, 420, 605

Momany, Y., Zaggia, S. R., Bonifacio, P., et al. 2004b, A\&A, 421, L29

Momany, Y., \& Zaggia, S. 2005, A\&A, 437, 339

Momany, Y., Held, E. V., Saviane, I., et al. 2005, A\&A, 439, 111

Monaco, L., Bellazzini, M., Ferraro, F. R., \& Pancino, E. 2003, ApJ, 597, L25

Monaco, L., Bellazzini, M., Bonifacio, P., et al. 2005, A\&A, 441, 141

Nakanishi, H., \& Sofue, Y. 2004, Milky Way Surveys: The Structure and Evolution of our Galaxy, ASP Conf. Ser., 317, 32

Newberg, H. J., Yanny, B., Rockosi, C., et al. 2002, ApJ, 569, 245

Newberg, H., \& Yanny, B., ASP Conf. Ser., Astrometry in the Age of the Next Generation of Large Telescopes 2005, ed. A. Monet, \& K. Seidelmann [arXiv: astro-ph/0502386]

Olano, C. A. 2004, A\&A, 423, 895

Oort, J. H., Kerr, F. J., \& Westerhout, G. 1958, MNRAS, 118, 379

Peñarrubia, J., Martínez-Delgado, D., Rix, H. W., et al. 2005, ApJ, 626, 128

Porcel, C., Battaner, E., \& Jimenez-Vicente, J. 1997, A\&A, 322, 103

Reshetnikov, V., \& Combes, F. 1998, A\&A, 337, 9

Robin, A., Reylé, C., Derrière, \& Picaud, S. 2003, A\&A, 409, 523

Rocha-Pinto, H., Majewski, S., Skrutskie, M., \& Crane, J. 2003, ApJ, 594, L115

Rocha-Pinto, H. J., Majewski, S. R., Skrutskie, M. F., Crane, J. D., \& Patterson, R. J. 2004, ApJ, 615, 732

Rocha-Pinto, H., Majewski, S. R., Skrutskie, M. F., \& Patterson, R. J. 2005, ApJ [arXiv: astro-ph/0504122]

Russeil, D. 2003, A\&A, 397, 133

Ruphy, S., Robin, A. C., Epchtein, N., et al. 1996, A\&A, 313, L21

Sánchez-Saavedra, M. L., Battaner, E., Guijarro, A., López-Corredoira, M., \& Castro-Rodríguez, N. 2003, A\&A, 399, 457

Sbordone, L., Bonifacio, P., Marconi, G., Zaggia, S., \& Buonanno, R. 2005, A\&A, 430, L13

Schlegel, D. J., Finkbeiner, D. P., \& Davis, M. 1998, ApJ, 500, 525

Schröder, S. E., Kaper, L., Lamers, H. J. G. L. M., \& Brown, A. G. A. 2004, A\&A, 428, 149

Snell, R. L., Carpenter, J. M., \& Heyer, M. H. 2002, ApJ, 578, 229

Soderblom, D. R., Nelan, E., Benedict, G. F., et al. 2005, AJ, 129, 1616

Taffoni, G., Mayer, L., Colpi, M., \& Governato, F. 2003, MNRAS, 341,434

Thilker, D. A., Bianchi, L., Boissier, S., et al. 2005, ApJ, 619, L79

Tsuchiya, T. 2002, New Astronomy, 7, 293

Tolstoy, E. 2000 [arXiv: astro-ph/0010028]

Tolstoy, E. 2005 [arXiv: astro-ph/0506481]

Vallée, J. P. 2005, AJ, 130, 569

Vig, S., Ghosh, S. K., \& Ojha, D. K. 2005, A\&A, 436, 867

Weinberg, M. D. 1998, MNRAS, 299, 499

Weaver, H., \& Williams, D. R. W. 1973, A\&AS, 8, 1

Willman, B., Dalcanton, J. J., Martinez-Delgado, D., et al. 2005, ApJ, 626, L85

Wouterloot, J., Brand, Burton, W., \& Kwee, K. 1990, A\&A, 230, 21 Wyse, R. F. G., \& Gilmore, G. 2005 [arXiv: astro-ph/0510025]

Yanny, B., Newberg, H. J., Grebel, E. K., et al. 2003, ApJ, 588, 824

Yusifov, I. 2004 [arXiv: astro-ph/0405517] 